\title{
Stabilization of saturated switching systems
}

\author{
Abdellah Benzaouia \\ University of Cadi Ayyad, Faculty of Science Semlalia \\ BP 2390, Marrakech, Morocco \\ benzaouia@ucam.ac.ma
}

\section{INTRODUCTION}

Switched systems are a class of hybrid systems encountered in many practical situations which involve switching between several subsystems depending on various factors. Generally, a switching system consists of a family of continuous-time subsystems and a rule that supervises the switching between them. This class of systems have numerous applications in the control of mechanical systems, the automotive industry, aircraft and air traffic control, switching power converters and many other fields. Two main problems are widely studied in the literature according to the classification given in (Blanchini and Savorgnan, 2006): The first one, which is the one solved in this work, looks for testable conditions that guarantee the asymptotic stability of a switching system under arbitrary switching rules, while the second is to determine a switching sequence that renders the switched system asymptotically stable (see (Liberzon and Morse, 1999) and the reference therein). Following the first approach, (Blanchini et al., 2009) investigate the problem of designing a switching compensator for a plant switching amongst a (finite) family of given configurations $\left(A_{i}, B_{i}, C_{i}\right)$.

A main problem which is always inherent to all dynamical systems is the presence of actuator saturations. Even for linear systems, this problem has been an active area of research for many years. Besides approaches using anti-windup techniques (Mulder et al., 2004) and model predictive controls (Camacho and Bordons, 2004), two main approaches have been developed in the literature: The first is the so-called positive invariance approach which is based on the design of controllers which work inside a region of linear behavior where saturations do not occur (see (Benzaouia and Burgat, 1988), (Benzaouia and Hmamed, 1993), (Blanchini, 1999) and the references therein). This approach has already being applied to a class of hybrid systems involving jumping parameters (Benzaouia and Boukas, 2002). It has also been used to design controllers for switching systems with constrained control under complete modelling taking into account reset functions at each switch and different system's dimension. The second approach, however allows saturations to take effect while guaranteeing asymptotic stability (see (Nguyen and Jabbari, 1999, 2000), (Tarbouriech et al., 2006), ( Hu et al., 2002)(Hu and Lin, 2002), (Benzaouia et al., 2006) and the references therein). The main challenge in these two approaches is to obtain large domains of initial states which ensures asymptotic stability for the system despite the presence of saturations (Gilbert and Tan 1991), (Benzaouia and Baddou, 1999), (Benzaouia et al., 2002), ( Hu et al., 2002).

The objective of this chapter is to present the available results in the literature for switching systems subject to actuator saturations. These results follow generally two ways: the first concerns the synthesis of non saturating controllers ( controllers working inside a large region 
of linear behavior where the saturations do not occur), while the second extends the results obtained for unsaturated switching systems by ( Mignone et al., 2000), ( Ferrari-Trecate et al., 2001) and (Daafouz et al., 2001, 2002) leading to saturating controllers ( controllers tolerating saturations to take effect). The second method was firstly used in (Benzaouia et al., 2004) with the use of a multiple Lyapunov function. However, only the intersection of all the corresponding level sets of the local functions was considered as a region of asymptotic stability of the switching system. This drawback is improved in (Benzaouia et al., 2006) and (Benzaouia et al., 2009a) by considering, for the first time, a large set of asymptotic stability composed by the union of all the level sets.

In this context, two main different sufficient conditions of asymptotic stability were obtained for switching systems subject to actuator saturations. Furthermore, these conditions were presented in the form of LMIs for the state feedback control case. A particular attention was given to the output feedback case which has an additive complexity due to the output equation. It was also shown that the LMIs obtained for computing controllers working inside a large region of linear behavior are less conservative.

The obtained results are then extended to uncertain switching system subject to actuator saturations as developed in (Benzaouia et al., 2009b) and (Benzaouia et al., 2009c) respectively. The uncertainty types considered in these two works are the polytopic one and the structured one. This second type of uncertainty was also studied, without saturation, in (Hetel et al., 2006). Thus, in this work (Benzaouia et al., 2009a), two directions are explored: the first concerns the synthesis of non saturating controllers, while the second direction deals with controllers tolerating saturations to take effect under polytopic uncertainties. For structured uncertainties studies in (Benzaouia et al., 2009b), the synthesis of the controller follows two different approaches, the first one deals firstly with the nominal system and then uses a test to check the asymptotic stability in presence of uncertainties while the second considers the global representation of the uncertain system.

\section{Stabilization of switching systems subject to actuator saturation}

\subsection{PROBLEM FORMULATION}

In this section, we give a more precise problem statement for the class of systems under consideration, namely, discrete-time switching linear systems with input saturation and state or output feedback. An equivalent description of such systems, based on the indicator function is also used in this work. The main results of this section are published in (Benzaouia et al., 2009a).

Thus, we consider systems described by:

$$
\begin{aligned}
x(t+1) & =A_{\alpha} x(t)+B_{\alpha} \operatorname{sat}(u(t)) \\
y(t) & =C_{\alpha} x(t)
\end{aligned}
$$

where $x \in \mathbb{R}^{n}$ is the state, $u \in \mathbb{R}^{m}$ is the control, $y \in \mathbb{R}^{p}$ is the output, sat(.) is the standard saturation function and $\alpha$ a switching rule which takes its values in the finite set $\mathcal{I}:=\{1, \ldots, N\}, t \in \mathbb{Z}_{+}$. The saturation function is assumed here to be normalized, $i$. $e$., $(|\operatorname{sat}(u)|=\min \{1,|u|\})$. Each subsystem $\alpha$ is called a mode.

Definition 2.1. (Lygeros et al., 1999) An hybrid time basis $\tau$ is an infinite or finite sequence of sets $I_{s}=\left\{t \in \mathbb{N}: t_{s} \leq t \leq \bar{t}_{s}\right\}$, with $\bar{t}_{s}=t_{s+1}$ for $s \in \mathcal{L}=\{0, \ldots, L\}$, and if $\operatorname{card}(\tau)=L+1<\infty$ then $\bar{t}_{L}$ can be finite or infinite. 
Throughout this chapter, it is assumed that:

- The switching system is stabilizable;

- Matrices $C_{\alpha}$ are of full rank;

- $t_{s+1} \geq t_{s}+1, \forall s \in \mathcal{L}$;

- the switching rule is not known a priori but $\alpha(t)$ is available at each $t$.

The third assumption ensures that at each time only one subsystem is active. The fourth assumption corresponds to practical implementations where the switched system is supervised by a discrete-event system or operator allowing for $\alpha(t)$ to be known in real time.

In this work, we are interested by the synthesis of stabilizing controllers for this class of hybrid systems subject to actuator saturation. We use a feedback control law as:

$$
u(t)=F_{\alpha} x(t)=K_{\alpha} y(t),
$$

and write the closed-loop system as

$$
\begin{aligned}
x(t+1) & =A_{\alpha} x(t)+B_{\alpha} \operatorname{sat}\left(F_{\alpha} x(t)\right) . \\
& =A_{\alpha} x(t)+B_{\alpha} \operatorname{sat}\left(K_{\alpha} y(t)\right)
\end{aligned}
$$

Upon introducing the indicator function:

$$
\xi(t)=\left[\xi_{1}(t), \ldots, \xi_{N}(t)\right]^{T}
$$

where $\xi_{i}(t)=1$ if the switching system is in mode $i$ and $\xi_{i}(t)=0$ if it is in a different mode, one can write the closed-loop system (3) as follows:

$$
\begin{aligned}
x(t+1) & =\sum_{i=1}^{N} \xi_{i}(t)\left[A_{i} x(t)+B_{i} \operatorname{sat}\left(F_{i} x(t)\right)\right] . \\
& =\sum_{i=1}^{N} \xi_{i}(t)\left[A_{i} x(t)+B_{i} \operatorname{sat}\left(K_{i} C_{i} x(t)\right)\right]
\end{aligned}
$$

\subsection{PRELIMINARY RESULTS}

In this section, we recall two results on which our work is based. Let $\alpha$ be fixed. Then System

(3) becomes a linear time-invariant system with input saturation given by:

$$
x(t+1)=A x(t)+B s a t(F x(t))
$$

Define the following subsets of $\mathbb{R}^{n}$ :

$$
\begin{gathered}
\varepsilon(P, \rho)=\left\{x \in \mathbb{R}^{n} \mid x^{T} P x \leq \rho\right\}, \\
\mathcal{L}(F)=\left\{x \in \mathbb{R}^{n}|| F_{l} x \mid \leq 1, l=1, \ldots, m\right\},
\end{gathered}
$$

with $P$ a positive definite matrix, $\rho>0$ and $F_{l}$ the $l$ th row of the matrix $F \in \mathbb{R}^{m \times n}$. Thus $\varepsilon(P, \rho)$ is an ellipsoid while $\mathcal{L}(F)$ is a polyhedral consisting of states for which the saturation does not occur.

Lemma 2.1. (Hu et al., 2002) For all $u \in \mathbb{R}^{m}$ and $v \in \mathbb{R}^{m}$ such that $\left|v_{l}\right|<1, l \in[1, m]$

$$
\operatorname{sat}(u) \in \operatorname{co}\left\{D_{s} u+D_{s}^{-} v, s \in[1, \eta]\right\} ; \eta=2^{m}
$$

where co denotes the convex hull. 
Consequently, there exist $\delta_{1} \geq 0, \ldots, \delta_{\eta} \geq 0$ with $\sum_{s=1}^{\eta} \delta_{s}=1$ such that,

$$
\operatorname{sat}(u)=\sum_{s=1}^{\eta} \delta_{s}\left[D_{s} u+D_{s}^{-} v\right]
$$

Here, $D_{s}$ is an $m$ by $m$ diagonal matrix with elements either 1 or 0 and $D_{s}^{-}=\mathbb{I}_{m}-D_{s}$. There are $2^{m}$ possible such matrices. One can also consult the work of (Benzaouia et al., 2006) for more details and other extensions to linear systems with both constraints on the control and the increment or rate of the control.

Consider the following autonomous switching system:

$$
x(t+1)=\sum_{i=1}^{N} \xi_{i}(t) A_{i} x(t)
$$

The use of the Lyapunov functions revealed two ways:

- The existence of a common Lyapunov function to the various subsystems guarantees the asymptotic stability of the switching system. In general, the search for such function is not always obvious (Shorten and Narenda, 1997, 1998).

- The multiple Lyapunov functions were introduced in (Branicky, 1998). They are considered as a strong tool in the analysis of the stability of the hybrid systems and in particular the switching systems.

Proposition 2.1. (Branicky, 1998) If there exists a multiple Lyapunov function $V(t, x), t \in I_{s}$, with $V: \mathbb{N} \times \mathbb{R}^{n} \longrightarrow \mathbb{R}_{+}$, such that the following hold:

- $\Delta V(t, x(t))<0$, for any time $t \in I_{s}$

- $V\left(t_{k}, x\left(t_{k}\right)\right)<V\left(t_{k-1}, x\left(t_{k-1}\right), \forall k \in \mathcal{L}\right.$

then, the switching autonomous system (11) is asymptotically stable.

We now recall a useful stability result for switching systems with no input saturations presented by many authors ( see (Mignone et al., 2000), ( Ferrari-Trecate et al., 2001) and ( Daafouz et al., 2001, 2002)) firstly used for linear time varying systems (Daafouz and Bernussou, 2001).

Theorem 2.1. The closed-loop switching system (11) is asymptotically stable at the origin if there exist $N$ symmetric and positive definite matrices $P_{1}, \ldots, P_{N}$ satisfying,

$$
A_{i}^{T} P_{j} A_{i}-P_{i}<0, \forall(i, j) \in \mathcal{I} \times \mathcal{I}
$$

A corresponding Lyapunov function for the system is then given by:

$$
V(t, x)=x^{T}(t)\left(\sum_{i=1}^{N} \xi_{i}(t) P_{i}\right) x(t)
$$

It is worth to note that function $V(t, x)$, which is a multiple Lyapunov function candidate involving matrices $P_{i}$, can be seen as a standard Lyapunov function candidate. It was the way followed by (Mignone et al., 2000) and (Daafouz et al., 2001, 2002). Further, condition (12) is equivalent, by using Schur complement to,

$$
\left[\begin{array}{cc}
P_{i} & A_{i}^{T} P_{j} \\
* & P_{j}
\end{array}\right]>0, \forall(i, j) \in \mathcal{I} \times \mathcal{I}
$$


where $*$ denotes the transpose of the off diagonal element of the LMI. Subsequently, we will need the following equivalent LMI representation of (14):

$$
\left[\begin{array}{cc}
X_{i} & X_{i} A_{i}^{T} \\
* & X_{j}
\end{array}\right]>0, \forall(i, j) \in \mathcal{I} \times \mathcal{I}
$$

where $X_{i}=P_{i}^{-1}$. By noting that inequality (12) is equivalent to:

$$
G_{i} A_{i}^{T} P_{j} A_{i} G_{i}^{T}-G_{i} P_{i} G_{i}^{T}<0, \forall(i, j) \in \mathcal{I} \times \mathcal{I}
$$

for any nonsingular matrix $G_{i}$. By using the fact that $\left(G_{i}-X_{i}\right)^{T} X_{i}^{-1}\left(G_{i}-X_{i}\right) \geq 0$, implies $G_{i} X_{i}^{-1} G_{i}^{T} \geq G_{i}+G_{i}^{T}-X_{i}$. The LMI (15) is also in turn equivalent to the following LMI, generally used to relax the previous one (Daafouz et al., 2001, 2002):

$$
\left[\begin{array}{cc}
G_{i}+G_{i}^{T}-X_{i} & G_{i}^{T} A_{i}^{T} \\
* & X_{j}
\end{array}\right]>0, \forall(i, j) \in \mathcal{I} \times \mathcal{I}
$$

where matrices $G_{i}$ called slack variables are nonsingular matrices and $X_{i}$ the positive definite matrices.

It is worth nothing that condition (12) has to be satisfied $\forall(i, j) \in \mathcal{I} \times \mathcal{I}$, in particular for $i=j$. This means that each mode is necessarily asymptotically stable and $V_{i}(t, x)=x^{T}(t) P_{i} x(t)$ is the associated Lyapunov function. Recall that a level set of the Lyapunov function $V(t, x)$ given by (13) and associated to the switching system (11) is given by the set $\varepsilon(P, \rho)$ defined by (7) with $P=\sum_{i=1}^{N} \xi_{i}(t) P_{i}$. This region of asymptotic stability is very difficult to construct. Nevertheless, an estimate can be obtained as large as possible as presented in the next section based on the union of the sub-level ellipsoid sets $\varepsilon\left(P_{i}, 1\right)$. Other type of level sets obtained with different Lyapunov functions for switched systems can be found in (Hu et al., 2006). A useful lemma is also recalled.

Lemma 2.2. Let $R, S$ and $\Gamma$ be matrices with appropriate dimension. Suppose $\Gamma^{T} \Gamma \leq \mathbb{I}$, then for any scalar $\lambda>0$, we have:

$$
R \Gamma S+S^{T} \Gamma^{T} R^{T} \leq \lambda R R^{T}+\lambda^{-1} S S^{T}
$$

\subsection{Analysis and synthesis of stabilizability}

In this section, the region of local asymptotic stability associated to the saturated switching system is firstly studied. The design of a stabilizing controller for the class of switching system with actuator saturation is then presented by following two ways, the first concerns controllers working inside the region of linear behavior where the saturations do not occur while the second is based on Lemma 2.1 tolerating saturations to occur.

\subsubsection{Region of asymptotic stability}

The link between the result of Theorem 2.1 and the level set (7) is not mentioned in (Mignone et al., 2000) nor in (Daafouz et al., 2001, 2002). The absence of saturation on the control of the switching systems in these works does not necessitate to take care with the level sets since the asymptotic stability is global. We present here after an interesting result on this subject inspired from the general class studied in (Benzaouia et al., 2007). 
Theorem 2.2. If there exist $N$ symmetric positive definite matrices $P_{1}, \ldots, P_{N}$ such that the inequalities (12) are satisfied then the set given by,

$$
\Omega=\bigcup_{i=1}^{N} \varepsilon\left(P_{i}, 1\right)
$$

is a level set of the multiple Lyapunov function $V(t, x)$ given by (13) with respect to the trajectories of the autonomous switching system (11).

Proof: Note that condition (12) for $i=j$ ensures that $\Delta V(t, x)<0$, for $t \in I_{s}, s \in \mathcal{L}$ (Daafouz et al., 2001, 2002). Now let $x\left(\bar{t}_{k-1}\right) \in \varepsilon\left(P_{i}, 1\right)$. What happens at the switching time $\bar{t}_{k-1}$ ?

For this, compute $x\left(t_{k}\right)$. With an identity reset function we have,

$$
x\left(t_{k}\right)=x\left(\bar{t}_{k-1}\right)
$$

The switch to the next region $\varepsilon\left(P_{j}, 1\right)$ will be seen an unit time after,

$$
\begin{aligned}
x\left(t_{k}+1\right) & =x\left(\bar{t}_{k-1}+1\right) \\
& =A_{i} x\left(\bar{t}_{k-1}\right)
\end{aligned}
$$

We have,

$$
x^{T}\left(t_{k}+1\right) P_{j} x\left(t_{k}+1\right)=x^{T}\left(\bar{t}_{k-1}\right)\left(A_{i}^{T} P_{j} A_{i}\right) x\left(\bar{t}_{k-1}\right)
$$

According to condition (12), one can obtain,

$$
x^{T}\left(t_{k}+1\right) P_{j} x\left(t_{k}+1\right)<x^{T}\left(\bar{t}_{k-1}\right) P_{i} x\left(\bar{t}_{k-1}\right)
$$

Since $x\left(\bar{t}_{k-1}\right) \in \varepsilon\left(P_{i}, 1\right)$, then, $x\left(t_{k}+1\right) \in \varepsilon\left(P_{j}, 1\right), \forall i, j \in \mathcal{I} \times \mathcal{I}$. This proves that $V\left(t_{k}, x\left(t_{k}\right)\right)<$ $V\left(t_{k-1}, x\left(t_{k-1}\right)\right), \forall k \in \mathcal{L}$. Consequently, function $V(t, x)$ is a multiple Lyapunov function of the switching system according to Proposition 2.1 and the set $\Omega$ is a level set associated to this function. It is worth to note that the same reasoning holds for $\varepsilon\left(P_{j}, \rho\right)$ for any positive scalar $\rho$.

Note that an important remark is to be done at this level: since the studied system is a switching one composed of $N$ subsystems (modes), the switching system can be initialized inside any level set $\varepsilon\left(P_{i}, 1\right)$, however, the initial mode to be selected is the corresponding mode $i$.

\subsubsection{State feedback control}

We assume that the state is available, so a state feedback control can be performed. The first result we present is a simple extension of known result given by (Gutman and Hagandar, 1985) for linear systems and concerns the synthesis of non saturating controllers ensuring that a large region of linear behavior is a region of asymptotic stability.

Theorem 2.3. If there exists $N$ symmetric matrices $X_{1}, \ldots, X_{N}$ and $N$ matrices $Y_{1}, \ldots, Y_{N}$ solutions of the following LMIs:

$$
\begin{gathered}
{\left[\begin{array}{cc}
X_{i} & \left(A_{i} X_{i}+B_{i} Y_{i}\right)^{T} \\
* & X_{j}
\end{array}\right]>0} \\
{\left[\begin{array}{cc}
1 & Y_{l i} \\
* & X_{i}
\end{array}\right]>0} \\
\forall(i, j) \in \mathcal{I} \times \mathcal{I}, \forall l \in[1, m]
\end{gathered}
$$


where $Y_{l i}$ is the lth row of matrix $Y_{i}$; then the switching system with saturations in closed-loop (3), with,

$$
F_{i}=Y_{i} X_{i}^{-1}, P_{i}=X_{i}^{-1}
$$

is asymptotically stable at the origin $\forall x_{0} \in \Omega$ and for any sequences of switching $\alpha(t)$.

Proof: In order to guarantee that the control by state feedback is always admissible, each sublevel set of the Lyapunov function has to be contained inside the polyhedral set where the saturations do not occur $\varepsilon\left(P_{i}, 1\right) \subset \mathcal{L}\left(F_{i}\right), \forall i \in \mathcal{I}$ (Gutman and Hagandar, 1985). Using (Boyd et al., 1994), this inclusion condition can also be transformed to the equivalent LMI (25) by letting $X_{i}=P_{i}^{-1}$ and $F_{i} X_{i}=Y_{i}$. Let $x(t) \in \Omega, \forall t \in I_{s}$. According to condition (25), the system in closed-loop (5) can be equivalently written as,

$$
x(t+1)=\sum_{i=1}^{N} \xi_{i}(t) A_{\mathcal{c}_{i}} x(t) .
$$

with, $A_{c_{i}}=A_{i}+B_{i} F_{i}$. The proof follows from the asymptotic stability conditions of the switching system given by Theorem 3.2, which is expressed equivalently by (15), with matrix $A_{c_{i}}$ instead of $A_{i}$. Further, Theorem 2.2 ensures that the set $\Omega$ is a set of asymptotic stability of the switching system with saturations in closed-loop (3).

To achieve a domain of attraction as large as possible, we can solve the following optimization problem:

$$
(P b .1):\left\{\begin{array}{c}
\sup _{\left(X_{i}, Y_{i}\right)} \operatorname{Trace}\left(X_{i}\right) \\
\text { s.t. }(24),(25) \\
i=1, \ldots, N
\end{array}\right.
$$

When this optimization problem is feasible, the obtained ellipsoid volumes are maximum with respect to the data of the system.

The obtained LMIs (24) of Theorem 2.1 are similar to those obtained in (Mignone et al., 2000) and (Ferrari-Trecate et al., 2001) for non saturated switching systems. The presence of saturation on the control in our problem leads to the additional LMIs (25), which will obviously restrict the set of solutions. Nevertheless, the associated large region of asymptotic stability $\Omega$ for the saturated switching system enables one to conclude that these LMIs are not conservative.

The second result we present concerns the synthesis of saturating controllers tolerating saturations to take effect inside a large region of asymptotic stability.

Theorem 2.4. If there exist symmetric positive definite matrices $P_{1}, \ldots, P_{N} \in \mathbb{R}^{n \times n}$ and matrices $H_{1}, \ldots, H_{N} \in \mathbb{R}^{m \times n}$ such that,

$$
\begin{array}{rr}
{\left[\begin{array}{cc}
P_{i} & {\left[A_{i}+B_{i}\left(D_{i s} F_{i}+D_{i s}^{-} H_{i}\right)\right]^{T} P_{j}} \\
* & P_{j}
\end{array}\right]>0,} \\
\forall(i, j) \in \mathcal{I} \times \mathcal{I}, \forall s \in[1, \eta]
\end{array}
$$

and,

$$
\varepsilon\left(P_{i}, 1\right) \subset \mathcal{L}\left(H_{i}\right), \forall i \in \mathcal{I}
$$

then, the closed-loop switching system (3) is asymptotically stable at the origin $\forall x_{0} \in \Omega$ and for all sequences of switching $\alpha(t)$. 
Proof: Assume that there exist $N$ matrices $H_{1}, \ldots, H_{N}$ and $N$ symmetric matrices $P_{1}, \ldots, P_{N}$ such that condition (28) and (29) are satisfied. Using the expression in (10) and rewriting System (3) as in (5) yields that:

$$
\begin{array}{r}
\operatorname{sat}\left(F_{i} x(t)\right)=\sum_{s=1}^{\eta} \delta_{i s}(t)\left[D_{i s} F_{i}+D_{i s}^{-} H_{i}\right] x(t) ; \\
\delta_{s i}(t) \geq 0, \sum_{s=1}^{\eta} \delta_{s i}(t)=1
\end{array}
$$

and, subsequently :

$$
\begin{aligned}
x(t+1) & =\sum_{s=1}^{\eta} \sum_{i=1}^{N} \xi_{i}(t) \delta_{i s}(t) A c_{i s} x(t) \\
A c_{i s} & :=A_{i}+B_{i}\left(D_{i s} F_{i}+D_{i s}^{-} H_{i}\right), \quad s \in[1, \eta]
\end{aligned}
$$

The rate of change of the Lyapunov function candidate (13) along the trajectories of (32) is given by:

$$
\begin{aligned}
& \Delta V(t, x(t))=x^{T}(t+1)\left(\sum_{j=1}^{N} \xi_{j}(t+1) P_{j}\right) x(t+1)-x^{T}(t)\left(\sum_{i=1}^{N} \xi_{i}(t) P_{i}\right) x(t)=x^{T}(t) \\
& {\left[\left(\sum_{s=1}^{\eta} \sum_{i=1}^{N} \xi_{i}(t) \delta_{i s}(t) A c_{i s}\right)^{T}\left(\sum_{j=1}^{N} \xi_{j}(t+1) P_{j}\right)\left(\sum_{s=1}^{\eta} \sum_{i=1}^{N} \xi_{i}(t) \delta_{i s}(t) A c_{i s}\right)-\sum_{i=1}^{N} \xi_{i}(t) P_{i}\right] x(t)}
\end{aligned}
$$

Let condition (28) be satisfied. At this level, for each $i$, multiply the $j=1, \ldots, N$ inequalities (28) by $\xi_{j}(t+1)$ and sum. Multiply the resulting $i=1, \ldots, N$ inequalities by $\xi_{i}(t)$ and sum. Multiply again the resulting $s=1, \ldots, \eta$ inequalities by $\delta_{i s}(t)$ and sum. As $\sum_{i=1}^{N} \xi_{i}(t)=$ $\sum_{j=1}^{N} \xi_{j}(t+1)=\sum_{s=1}^{\eta} \delta_{i s}(t)=1$, one gets:

$$
\left[\begin{array}{cc}
\sum_{i=1}^{N} \xi_{i}(t) P_{i} & \Gamma \sum_{j=1}^{N} \xi_{j}(t+1) P_{j} \\
* & \sum_{j=1}^{N} \xi_{j}(t+1) P_{j}
\end{array}\right]>0,
$$

with, $\Gamma=\left[\sum_{s=1}^{\eta} \sum_{i=1}^{N} \xi_{i}(t) \delta_{i s}(t) A c_{i s}\right]^{T}$. The use of Schur complement allows us to write condition (34) under the equivalent form,

$$
\begin{array}{r}
\left(\sum_{s=1}^{\eta} \sum_{i=1}^{N} \xi_{i}(t) \delta_{i s}(t) A c_{i s}\right)^{T}\left(\sum_{j=1}^{N} \xi_{j}(t+1) P_{j}\right)\left(\sum_{s=1}^{\eta} \sum_{i=1}^{N} \xi_{i}(t) \delta_{i s}(t) A c_{i s}\right)-\sum_{i=1}^{N} \xi_{i}(t) P_{i}<0 \\
\forall(i, j) \in \mathcal{I} \times \mathcal{I}, \forall s \in[1, \eta]
\end{array}
$$

Letting $\lambda$ be the largest eigenvalue among all the above matrices, we obtain that

$$
\Delta V(t, x(t)) \leq \lambda x^{T}(t) x(t)<0
$$

which ensures the desired result. Noting that condition (28) is also satisfied for $i=j$, this implies that each set $\varepsilon\left(P_{i}, 1\right)$ is a contractively invariant set for the corresponding subsystem. 
Further, by taking account of condition (29), one can guarantee that for every $x_{o} \in \varepsilon\left(P_{i}, 1\right) \subset$ $\mathcal{L}\left(H_{i}\right)$, each subsystem is asymptotically stable at the origin. Besides, following Theorem 2.2, conditions (28)-(29) also allow for a state belonging, before the switch, to a set $\varepsilon\left(P_{i}, 1\right) \subset$ $\mathcal{L}\left(H_{i}\right)$, if a switch occurs at any time $t_{k}$, that the switch will handle the state to the desired set $\varepsilon\left(P_{j}, 1\right) \subset \mathcal{L}\left(H_{j}\right)$. That means that the set $\Omega$ is a set of asymptotic stability of the switching system.

This stability result can be used for control synthesis as follows.

Theorem 2.5. If there exists $N$ symmetric matrices $X_{1}, \ldots, X_{N}$ and $2 N$ matrices $Y_{1}, \ldots, Y_{N}$ and $Z_{1}, \ldots, Z_{N}$ solutions of the following LMIs:

$$
\begin{gathered}
{\left[\begin{array}{cc}
X_{i} & \left(A_{i} X_{i}+B_{i} D_{i s} Y_{i}+B_{i} D_{i s}^{-} Z_{i}\right)^{T} \\
* & X_{j}
\end{array}\right]} \\
{\left[\begin{array}{cc}
1 & Z_{l i} \\
* & X_{i}
\end{array}\right]>0,} \\
\forall(i, j) \in \mathcal{I} \times \mathcal{I}, \forall s \in[1, \eta], \forall l \in[1, m]
\end{gathered}
$$

then the switching system with saturations in closed-loop (3), with,

$$
F_{i}=Y_{i} X_{i}^{-1}, H_{i}=Z_{i} X_{i}^{-1}, P_{i}=X_{i}^{-1}
$$

is asymptotically stable at the origin $\forall x_{0} \in \Omega$ and for any sequences of switching $\alpha(t)$.

Proof: The Inequalities (28) can be transformed equivalently by Schur complement to the following:

$$
P_{j}\left[A_{i}+B_{i}\left(D_{i s} F_{i}+D_{i s}^{-} H_{i}\right)\right] P_{i}^{-1}\left[A_{i}+B_{i}\left(D_{i s} F_{i}+D_{i s}^{-} H_{i}\right)\right]^{T} P_{j}-P_{j}<0
$$

By pre and post-multiplying the latter by $P_{j}^{-1}$, it follows:

$$
\left[A_{i}+B_{i}\left(D_{i s} F_{i}+D_{i s}^{-} H_{i}\right)\right] P_{i}^{-1}\left[A_{i}+B_{i}\left(D_{i s} F_{i}+D_{i s}^{-} H_{i}\right)\right]^{T}-P_{j}^{-1}<0
$$

Pose $X_{i}=P_{i}^{-1}$, inequality (41) can then be rewritten as,

$$
\left[A_{i}+B_{i}\left(D_{i s} F_{i}+D_{i s}^{-} H_{i}\right)\right] X_{i}\left[A_{i}+B_{i}\left(D_{i s} F_{i}+D_{i s}^{-} H_{i}\right)\right]^{T}-X_{j}<0,
$$

that is

$$
\left[A_{i} X_{i}+B_{i}\left(D_{i s} F_{i} X_{i}+D_{i s}^{-} H_{i} X_{i}\right)\right] X_{i}^{-1}\left[A_{i} X_{i}+B_{i}\left(D_{i s} F_{i} X_{i}+D_{i s}^{-} H_{i} X_{i}\right)\right]^{T}-X_{j}<0 .
$$

The use of the Schur complement a second time leads to:

$$
\left[\begin{array}{cc}
X_{i} & \left(A_{i} X_{i}+B_{i} D_{i s} F_{i} X_{i}+B_{i} D_{i s}^{-} H_{i} X_{i}\right)^{T} \\
* & X_{j}
\end{array}\right]>0
$$

By letting $F_{i} X_{i}=Y_{i}$ and $H_{i} X_{i}=Z_{i}$, the LMI (37) follows readily. Using (Boyd et al., 1994), the inclusion condition (29) can also be transformed to the equivalent LMI (38). 
To obtain larger ellipsoid domains $\varepsilon\left(P_{i}, 1\right)$, we can apply the following optimization problem:

$$
(P b .2):\left\{\begin{array}{c}
\sup _{\left(X_{i}, Y_{i}, Z_{i}\right)} \operatorname{Trace}\left(X_{i}\right) \\
\text { s.t. }(37),(38), \\
i=1, \ldots, N
\end{array}\right.
$$

When this optimization problem is feasible, the obtained ellipsoid volumes are maximum with respect to the data of the system.

Remark 2.1. The results presented in this section can be extended easily to level sets $\varepsilon\left(P_{i}, \rho_{i}\right)$ where the scalars $\rho_{i}, i=1, \ldots, N$ can be a priori fixed, by using the variables $X_{i}=P_{i} / \rho_{i}$ in the LMIs (24)(25) and (37)-(38).

Commennt 2.1. The resolution of the LMIs (37) for $s \in[1, \eta]$, may be very restrictive. One can relax this resolution by accepting controllers working in a region of linear behavior where the saturations do not occur. The obtained results in this work, where the switching system is with saturated control, are a set of $\left(2^{m} \times N^{2}+N m\right)$ LMIs for computing stabilizing controllers working inside the saturated regions $\mathcal{L}\left(H_{i}\right)$ or only a set of $\left(N^{2}+N m\right)$ LMIs for computing stabilizing controllers working inside regions of linear behavior $\mathcal{L}\left(F_{i}\right)$. Note also that the LMIs of Theorem 2.3 can be obtained from LMIs of Theorem 2.5 by letting $D_{i s}=\mathbb{I}$ and $D_{i s}^{-}=0$.

Example 2.1. Consider a numerical switching discrete-time system with saturation specified by the two subsystems:

$$
A_{1}=\left[\begin{array}{cc}
-0.7 & 1 \\
-0.5 & -1.5
\end{array}\right] ; B_{1}=\left[\begin{array}{l}
1 \\
0
\end{array}\right] ; A_{2}=\left[\begin{array}{cc}
0.9 & -1 \\
1.7 & -1.5
\end{array}\right] ; B_{2}=\left[\begin{array}{c}
0 \\
-1
\end{array}\right] .
$$

We have to solve 5 LMIs with 4 variables to compute controllers working inside a region of linear behavior. The use of the Matlab LMI Toolbox to check our conditions leads to the following results without using the optimization problem (Pb.1).

$$
\begin{gathered}
P_{1}=\left[\begin{array}{cc}
7.3328 & 18.7125 \\
18.7125 & 55.9711
\end{array}\right] ; F_{1}=\left[\begin{array}{ll}
1.2244 & 0.7535
\end{array}\right] ; A c_{1}=\left[\begin{array}{cc}
0.5244 & 1.7535 \\
-0.5000 & -1.5000
\end{array}\right] ; \\
P_{2}=\left[\begin{array}{cc}
4.8237 & -5.1001 \\
-5.1001 & 5.6715
\end{array}\right] ; F_{2}=\left[\begin{array}{ll}
1.7559 & -1.5699
\end{array}\right] ; A c_{2}=\left[\begin{array}{cc}
0.9000 & -1.0000 \\
-0.0559 & 0.0699
\end{array}\right] ;
\end{gathered}
$$

Figure 1 presents the two ellipsoid sets of invariance and contractivity $\varepsilon\left(P_{i}, 1\right)$ together with the sets of linear behavior $\mathcal{L}\left(F_{i}\right)$ computed with LMIs (24)-(25).

For the same example, the use of the Matlab LMI Toolbox to check conditions (37)-(38) leads to the following results by using the optimization problem (Pb.2) with 10 LMIs and 6 variables.

$$
\begin{aligned}
P_{1} & =\left[\begin{array}{cc}
7.4032 & 18.1841 \\
18.1841 & 56.6345
\end{array}\right] ; H_{1}=\left[\begin{array}{ll}
1.2534 & 0.8569
\end{array}\right] ; F_{1}=\left[\begin{array}{ll}
1.2641 & 0.8526
\end{array}\right] ; \\
A c_{1} & =\left[\begin{array}{cc}
0.5641 & 1.8526 \\
-0.5000 & -1.5000
\end{array}\right] ; \\
P_{2} & =\left[\begin{array}{cc}
4.5202 & -4.3908 \\
-4.3908 & 6.5909
\end{array}\right] ; H_{2}=\left[\begin{array}{ll}
1.7693 & -1.5845
\end{array}\right] ; F 2=\left[\begin{array}{ll}
1.7697 & -1.5836
\end{array}\right] ; \\
A c_{2} & =\left[\begin{array}{cc}
0.9000 & -1.0000 \\
-0.0697 & 0.0836
\end{array}\right] ;
\end{aligned}
$$




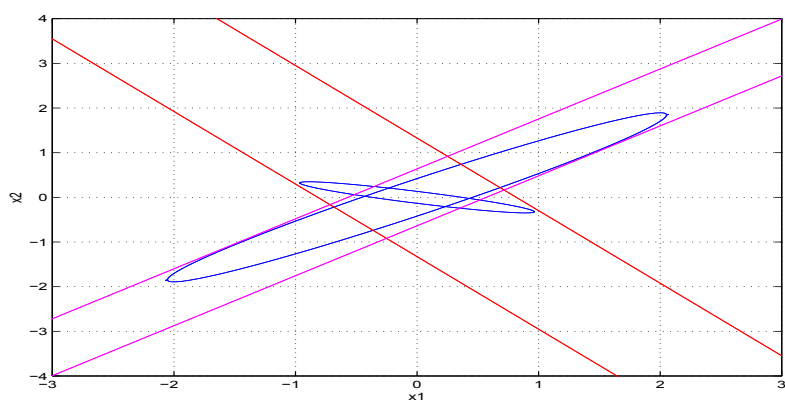

Fig. 1. The ellipsoids sets of invariance and contractivity for the switching discrete-time linear system computed with LMIs (24)-(25).

Note that the optimal values of the optimization problem (Pb.2) are given by $\operatorname{Trace}\left(P_{1}^{-1}\right)=0.7227$ and $\operatorname{Trace}\left(P_{2}^{-1}\right)=1.0568$. Figure 2 presents the two ellipsoid sets of invariance and contractivity

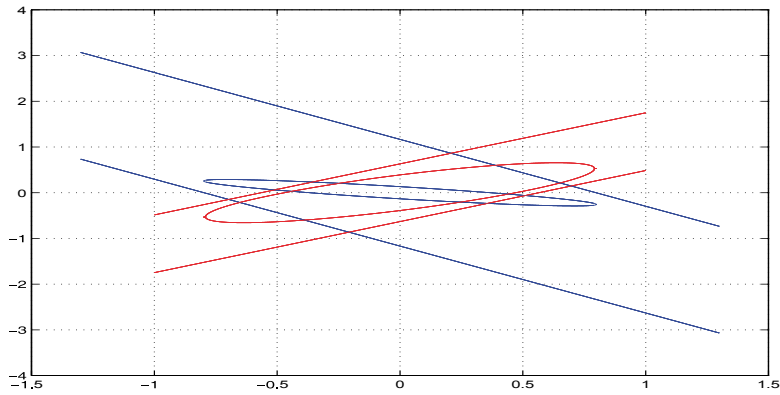

Fig. 2. The ellipsoids sets of invariance and contractivity for the switching discrete-time linear system computed with LMIs (37)-(38) and (Pb.2).

$\varepsilon\left(P_{i}, 1\right)$ together with the set of saturations $\mathcal{L}\left(H_{i}\right)$ computed with (Pb.2).

Comparing the results obtained by LMIs (37)-(38) and LMIs (24)-(25), one can note that the matrices $H_{i}$ are very closed up to matrices $F_{i}$ obtained with LMIs (37)-(38), that is, $\mathcal{L}\left(H_{i}\right) \cong$ $\mathcal{L}\left(F_{i}\right)$. This means that in the case of this example, the saturations allowed by this technique are not very important. Recall that inside the sets $\mathcal{L}\left(F_{i}\right)$ no saturations occur. Furthermore, the results obtained with LMIs (24)-(25) are less conservative. This is due to the fact that among the number of LMIs to be solved in (37)-(38), one find the LMIs (24)-(25).

\subsubsection{Output feedback control}

Static output-feedback control plays a very important role in control applications: The purpose is to design controllers such that the resulting closed-loop system is asymptotically stable without using any reconstruction method of the unavailable states. In this section, we begin by the synthesis of the saturating controllers since the non saturating ones can be derived as a particular case. The synthesis of the stabilizing controller by output feedback control for the class of switching system with actuator saturation is presented by applying 
the results of Theorem 2.4.

Theorem 2.6. If there exist symmetric matrices $X_{i}$, matrices $V_{i}, Y_{i}$ and $Z_{i}$ solutions of the following LMIs:

$$
\begin{gathered}
{\left[\begin{array}{cc}
X_{i} & \left(A_{i} X_{i}+B_{i} D_{i s} Y_{i} C_{i}+B_{i} D_{i s}^{-} Z_{i}\right)^{T} \\
* & X_{j}
\end{array}\right]>0,} \\
\left.\quad \begin{array}{cc}
1 & Z_{i l} \\
* & X_{i}
\end{array}\right]>0, \\
V_{i} C_{i}=C_{i} X_{i}
\end{gathered}
$$

then the closed-loop saturated switching system (3) with

$$
K_{i}=Y_{i} V_{i}^{-1}, H_{i}=Z_{i} X_{i}^{-1}, P_{i}=X_{i}^{-1}
$$

is asymptotically stable $\forall x_{0} \in \Omega$ and for all switching sequences $\alpha(k)$.

Proof: The same development as (40)-(42) while replacing $F_{i}$ by $K_{i} C_{i}$, can be followed to obtain,

$$
\left[\begin{array}{cc}
X_{i} & \left(A_{i} X_{i}+B_{i} D_{i s} K_{i} C_{i} X_{i}+B_{i} D_{i s}^{-} H_{i} X_{i}\right)^{T} \\
* & X_{j}
\end{array}\right]>0 .
$$

According to Equation (45), we have $K_{i} C_{i} X_{i}=K_{i} V_{i} C_{i}$. By letting $K_{i} V_{i}=Y_{i}$ and $H_{i} X_{i}=Z_{i}$, the LMI (43) follows together with relations (46). Similarly, the inclusion condition (29) can also be transformed to the equivalent LMI (44). Finally, the inequality (43) ensures that the obtained solutions $X_{i}$ are positive definite while equalities (45) guarantee that matrices $V_{i}$ are nonsingular.

It is worth noting that the state feedback follows readily from Theorem 2.6 by letting $C_{i}=\mathbb{I}_{m}$. In this case, $V_{i}=X_{i}$.

The LMI (43), relating matrices $C_{i}$ to matrix $X_{i}$ by means of Equation (45), can be relaxed by using the LMI (17), where the new variables $G_{i}$ are related to matrices $C_{i}$ instead of the matrix $X_{i}$.

Corollary 2.1. If there exist symmetric matrices $X_{i}$, matrices $G_{i}, V_{i}, Y_{i}$ and $Z_{i}$ solutions of the following LMIs:

$$
\begin{aligned}
{\left[\begin{array}{rr}
G_{i}+G_{i}^{T}-X_{i} & \Psi_{i s}^{T} \\
* & X_{j}
\end{array}\right] } & >0, \\
{\left[\begin{array}{rr}
1, & Z_{i l} \\
* & G_{i}+G_{i}^{T}-X_{i}
\end{array}\right] } & >0, \\
V_{i} C_{i} & =C_{i} G_{i}
\end{aligned}
$$

where $\Psi_{i s}=\left(A_{i} G_{i}+B_{i} D_{i s} Y_{i} C_{i}+B_{i} D_{i s}^{-} Z_{i}\right)$, then the closed-loop saturated switching system (3) with

$$
K_{i}=Y_{i} V_{i}^{-1}, H_{i}=Z_{i} G_{i}^{-1}, P_{i}=X_{i}^{-1}
$$

is asymptotically stable $\forall x_{0} \in \Omega$ and all switching sequences $\alpha(k)$. 
Proof: The proof uses the equivalent LMI (17) and is similar to that of Theorem 2.6. However, the inclusion condition $\varepsilon\left(P_{i}, 1\right) \subset \mathcal{L}\left(H_{i}\right) \quad \forall i \in \mathcal{I}$ holds if $1-H_{i l} X_{i} H_{i l}^{T}>0, \forall l \in[1, m]$, which is equivalent to,

$1-\left(H_{i} G_{i}\right)_{l}\left(G_{i}^{T} X_{i}^{-1} G_{i}\right)^{-1}\left(H_{i} G_{i}\right)_{l}^{T}>0$. That is, by virtue of (50) 1 $\left(Z_{i l}\right)\left(G_{i}^{T} X_{i}^{-1} G_{i}\right)^{-1}\left(Z_{i l}\right)^{T}>0$. Since, $\left(G_{i}-X_{i}\right)^{T} X_{i}^{-1}\left(G_{i}-X_{i}\right) \geq 0$, then $G_{i} X_{i}^{-1} G_{i}^{T} \geq$ $G_{i}+G_{i}^{T}-X_{i}$. It follows that, $1-Z_{i l}\left(G_{i}+G_{i}^{T}-X_{i}\right) Z_{i l}^{T}>0$ sufficient to have $\varepsilon\left(P_{i}, 1\right) \subset \mathcal{L}\left(H_{i}\right)$. By Schur complement, the LMI (48) is obtained.

To achieve a domain of attraction as large as possible, we can solve similar optimization problem as for state feedback control.

Another way to deal with the problem by relaxing the more constraining relations (45) of Theorem 2.6, especially in MIMO case, is presented by the following result which uses the same idea as in (El Ghaoui et al., 1997) and (Chadli et al., 2002).

Theorem 2.7. If there exist symmetric matrices $X_{i}$ and $S_{i}$, matrices $Y_{i}$ and $Z_{i}$ solutions of the following LMIs:

$$
\begin{aligned}
& {\left[\begin{array}{cc}
X_{i} & \left(A_{i}+B_{i} D_{i s} Y_{i} C_{i}+B_{i} D_{i s}^{-} Z_{i}\right)^{T} \\
* & S_{j}
\end{array}\right]>0,} \\
& {\left[\begin{array}{ll}
1 & Z_{i l} \\
* & X_{i}
\end{array}\right]>0,} \\
& {\left[\begin{array}{cc}
X_{i} & \mathbb{I} \\
* & S_{i}
\end{array}\right] \geq 0} \\
& \forall(i, j) \in \mathcal{I}^{2}, \quad \forall s \in[1, \eta], \quad \forall l \in[1, m]
\end{aligned}
$$

such that Trace $\left(X_{i} S_{i}\right)=n$, then the closed-loop saturated switching system (3) with

$$
K_{i}=Y_{i}, H_{i}=Z_{i}, P_{i}=X_{i}
$$

is asymptotically stable $\forall x_{0} \in \Omega$ and all switching sequences $\alpha(k)$.

Proof: The inequality (28) can be equivalently transformed via the Schur Complement to the following:

$$
\left[A_{i}+B_{i}\left(D_{i s} K_{i} C_{i}+D_{i s}^{-} H_{i}\right)\right]^{T} P_{j}\left[A_{i}+B_{i}\left(D_{i s} K_{i} C_{i}+D_{i s}^{-} H_{i}\right)\right]-P_{i}<0
$$

The use of the Schur complement a second time leads to

$$
\left[\begin{array}{cc}
P_{i} & \left(A_{i}+B_{i} D_{i s} K_{i} C_{i}+B_{i} D_{i s}^{-} H_{i}\right)^{T} \\
* & P_{j}^{-1}
\end{array}\right]>0
$$

By letting $X_{i}=P_{i}, S_{j}=P_{j}^{-1}, Y_{i}=K_{i}$ and $Z_{i}=H_{i}$, the LMI (51) follows together with relations (54). The inclusion condition (29) can also be transformed to the equivalent LMI (52) (Boyd et al., 1994): $H_{i l} P_{i}^{-1} H_{i l}^{T} \leq 1$ can be rewritten by Schur complement, while using $Z_{i}=H_{i}$ as (52). Note that the LMI (51) ensures that the obtained solutions $X_{i}$ and $S_{i}$ are positive definite. Finally, the LMI (53) is equivalent to $S_{i} X_{i} \geq \mathbb{I}$. 
This result presents the advantage of computing directly the matrices $K_{i}$ and $H_{i}$. Nevertheless, an optimization problem must be solved to achieve $X_{i} S_{i} \simeq \mathbb{I}$ by minimizing iteratively the trace of matrix $X_{i} S_{i}$ using an algorithm presented in (El Ghaoui et al., 1997). This heuristic is based on a linear approximation of $\operatorname{Tr}\left(X_{i} S_{i}\right)$ by $\operatorname{Tr}\left(X_{0} S_{i}+S_{0} X_{i}\right)$ where $X_{0}$ and $S_{0}$ are particular solutions of the LMI constraints (51), (52) and (53).

$$
(P b .3):\left\{\begin{array}{c}
\min _{\left(S_{j}, X_{i}, Y_{i}, Z_{i}\right)} \operatorname{Trace}\left(X_{i} S_{i}\right) \\
\text { s.t. }(51),(52),(53) \\
i, j=1, \ldots, N
\end{array}\right.
$$

An output feedback non saturating controller can be obtained, as noted in Comment 2.1, as a particular case of the saturating controller results by letting $D_{i s}=\mathbb{I}$ and $D_{i s}^{-}=0$. It is also obvious that the inclusion condition becomes $\varepsilon\left(P_{i}, 1\right) \subset \mathcal{L}\left(K_{i} C_{i}\right)$. The following results are then directly obtained.

Corollary 2.2. If there exist symmetric matrices $X_{i}$, matrices $V_{i}$ and $Y_{i}$ solutions of the following LMIs:

$$
\begin{aligned}
& {\left[\begin{array}{cc}
X_{i} & \left(A_{i} X_{i}+B_{i} Y_{i} C_{i}\right)^{T} \\
* & X_{j}
\end{array}\right]>0,} \\
& {\left[\begin{array}{cc}
1 & \left(Y_{i} C_{i}\right)_{l} \\
* & X_{i}
\end{array}\right]>0} \\
& V_{i} C_{i}=C_{i} X_{i} \\
& \forall(i, j) \in \mathcal{I}^{2}, \forall l \in[1, m]
\end{aligned}
$$

then the closed-loop saturated switching system (3) with

$$
K_{i}=Y_{i} V_{i}^{-1}, P_{i}=X_{i}^{-1}
$$

is asymptotically stable $\forall x_{0} \in \Omega$ and for all switching sequences $\alpha(k)$.

Proof: This proof can be obtained easily by letting $D_{i s}=\mathbb{I}$ and $D_{i s}^{-}=0$ in Theorem 2.6. However, the inclusion condition $\varepsilon\left(P_{i}, 1\right) \subset \mathcal{L}\left(K_{i} C_{i}\right)$ leads to $\left(K_{i} C_{i}\right)_{l} P_{i}^{-1}\left(K_{i} C_{i}\right)_{l}^{T} \leq 1$ which is equivalent to,

$$
\left(K_{i} C_{i} P_{i}^{-1}\right)_{l} P_{i}\left(K_{i} C_{i} P_{i}^{-1}\right)_{l}^{T} \leq 1,
$$

by using (59), $\left(K_{i} V_{i} C_{i}\right)_{l} X_{i}^{-1}\left(K_{i} V_{i} C_{i}\right)_{l}^{T} \leq 1$, can be rewritten by Schur complement, while using $Y_{i}=K_{i} V_{i}$ as (58).

In a similar way, the result of Theorem 2.7 can be applied for designing non saturating controls by letting $D_{i s}=\mathbb{I}$ and $D_{i s}^{-}=0$.

Example 2.2. In order to illustrate these results, consider a SISO saturated switching discrete-time system with two modes given by the following matrices:

$$
\begin{gathered}
A_{1}=\left[\begin{array}{ll}
1 & 1 \\
0 & 1
\end{array}\right] ; B_{1}=\left[\begin{array}{c}
10 \\
5
\end{array}\right] ; C_{1}=\left[\begin{array}{ll}
1 & 1
\end{array}\right] \\
A_{2}=\left[\begin{array}{cc}
0 & -1 \\
0.0001 & 1
\end{array}\right] ; B_{2}=\left[\begin{array}{c}
0.5 \\
-2
\end{array}\right] ; C_{2}=\left[\begin{array}{ll}
2 & 3
\end{array}\right]
\end{gathered}
$$


For this example with $n=2, m=p=1$ and $N=2$, we have to solve 13 LMIs with 9 variables by using the LMIs of Corollary 2.1. Let the scalar $\rho=1$. The use of the Matlab LMI Toolbox yields the following results:

$$
\begin{aligned}
P_{1} & =\left[\begin{array}{ll}
0.0016 & 0.0006 \\
0.0006 & 0.0025
\end{array}\right] ; H_{1}=\left[\begin{array}{cc}
-0.0196 & -0.0479
\end{array}\right] ; \\
K_{1} & =-0.0743 ; A c_{1}=\left[\begin{array}{cc}
0.2574 & 0.2574 \\
-0.3713 & 0.6287
\end{array}\right] ; \\
P_{2} & =\left[\begin{array}{ll}
0.0010 & 0.0004 \\
0.0004 & 0.0027
\end{array}\right] ; H_{2}=\left[\begin{array}{cc}
0.000007 & 0.0483
\end{array}\right] ; \\
K_{2} & =0.0347 ; A c_{2}=\left[\begin{array}{cc}
0.0615 & -0.9077 \\
-0.2460 & 0.6309
\end{array}\right]
\end{aligned}
$$

The main conditions (12) are also satisfied:

$$
\begin{gathered}
A c_{1}^{T} P_{2} A c_{1}-P_{1}=10^{-3}\left[\begin{array}{ll}
-0.0012 & -0.0011 \\
-0.0011 & -0.0013
\end{array}\right] ; \\
A c_{2}^{T} P_{1} A c_{2}-P_{2}=10^{-3}\left[\begin{array}{ll}
-0.0008 & -0.0007 \\
-0.0007 & -0.0011
\end{array}\right]
\end{gathered}
$$

Figure 3 shows the level set as the union of two ellipsoid sets of invariance and contractivity for

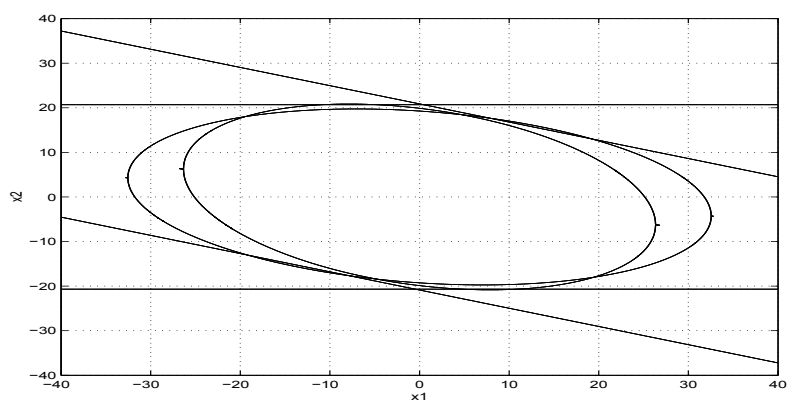

Fig. 3. The level set as the union of two ellipsoid sets of invariance and contractivity for the switching discrete-time linear system obtained by Corollary 2.1.

the switching discrete-time linear system obtained by Corollary 2.1. Each ellipsoid set is contained inside the set of admissible saturations $\mathcal{L}\left(H_{i}\right)$. Inside this set, the asymptotic stability of the saturated switching system is guaranteed for any arbitrary switch from any linear subsystem to another. This SISO example is studied only in order to illustrate the results of this work by plotting the level set in the plane. The use of Theorem 2.7 leads to the following results for $\rho=1$ :

$$
\begin{gathered}
P_{1}=\left[\begin{array}{ll}
1.0961 & 0.0848 \\
0.0848 & 1.0290
\end{array}\right] ; P_{2}=\left[\begin{array}{cc}
1.5366 & 0.1255 \\
0.1255 & 1.4821
\end{array}\right] ; \\
S_{1}=\left[\begin{array}{cc}
0.6553 & -0.0555 \\
-0.0555 & 0.6794
\end{array}\right] ; S_{2}=\left[\begin{array}{cc}
0.9182 & -0.0756 \\
-0.0756 & 0.9781
\end{array}\right] ;
\end{gathered}
$$


$H_{1}=\left[\begin{array}{ll}-0.0786 & -0.1208\end{array}\right] ; K_{1}==-0.1007 ; H_{2}=\left[\begin{array}{cc}0.00004 & 0.5473\end{array}\right] ; K 2=0.1812$

The level set obtained with this approach is plotted in Figure 4 together with few trajectories.
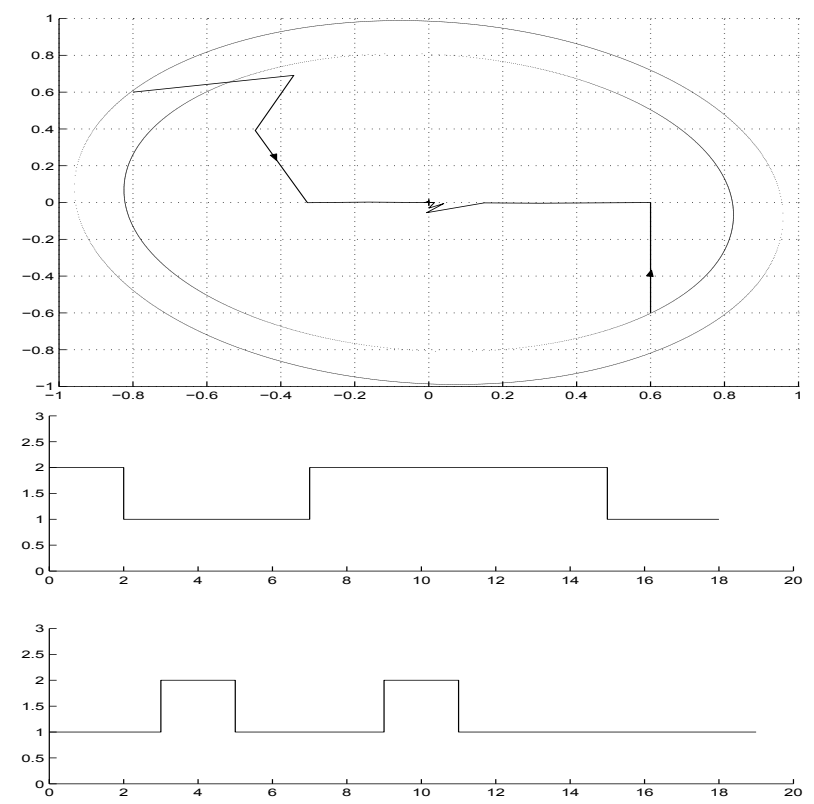

Fig. 4. The level set as the union of two ellipsoid sets of invariance and contractivity for the switching discrete-time linear system obtained by Theorem 2.7 and the corresponding switching sequences.

One can notice that the obtained level sets with Corollary2.1 are the most larger. One can conclude, via this example, that the result of Corollary 2.1 is the less conservative among all the other results due to the introduced slack variables $G_{i}$. Combining the comparisons made for state feedback control and output feedback control, one can expect that the introduction of slack variables in the Corollary 2.2 can lead to the more less conservative results.

In this section, two main different sufficient conditions of asymptotic stability are obtained for switching discrete-time linear systems subject to actuator saturations for each case: state feedback and output feedback control. The first allows the synthesis of stabilizing controllers inside a large region of linear behavior while the second applies the idea of Lemma 2.1 which rewrites the saturation function under a combination of $2^{m}$ elements to obtain stabilizing controllers tolerating saturations to take effect.

\section{Stabilization of saturated switching systems with polytopic uncertainties}

The objective of this section is to extend the results of (Benzaouia et al., 2006) to uncertain switching system subject to actuator saturations. The uncertainty type considered in this work is the polytopic one. This type of uncertainty was also studied, without saturation, in (Hetel et al., 2006). Thus, in this work, two directions are explored: the first concerns the synthesis of non saturating controllers, while the second direction deals with controllers 
tolerating saturations to take effect under polytopic uncertainties. The main results of this section are published in (Benzaouia et al., 2009b).

\subsection{Problem presentation}

Let us consider the uncertain saturated switching discrete-time linear system described by:

$$
\left\{\begin{array}{l}
x_{t+1}=A_{\alpha}\left(q_{\alpha}(t)\right) x_{t}+B_{\alpha}\left(q_{\alpha}(t)\right) \operatorname{sat}\left(u_{t}\right) \\
y_{t}=C_{\alpha}\left(q_{\alpha}(t)\right) x_{t}
\end{array}\right.
$$

where $x_{t} \in \mathbb{R}^{n}, u_{t} \in \mathbb{R}^{m}, y_{t} \in \mathbb{R}^{p}$ are the state, the input and the output respectively, sat(.) is the standard saturation (assumed here to be normalized, i.e., $\left|\operatorname{sat}\left(u_{t}\right)\right|=\min \left(1,\left|u_{t}\right|\right)$ ), function $\alpha(t): \mathbb{N} \longmapsto \mathcal{I}$ is a switching rule taking its values $\alpha(t)=i$ in the finite set $\mathcal{I}=\{1, \ldots, N\}$ and $q_{\alpha}(t) \in \Gamma_{\alpha} \subset \mathbb{R}^{d_{\alpha}}$ are the bounded uncertainties that affect the system parameters in such a way that

$$
\begin{aligned}
& A_{\alpha}\left(q_{\alpha}(t)\right)=A_{\alpha}+\sum_{h=1}^{d_{\alpha}} A_{\alpha h} q_{\alpha h}(t) \\
& B_{\alpha}\left(q_{\alpha}(t)\right)=B_{\alpha}+\sum_{h=1}^{d_{\alpha}} B_{\alpha h} q_{\alpha h}(t) \\
& C_{\alpha}\left(q_{\alpha}(t)\right)=C_{\alpha}+\sum_{h=1}^{d_{\alpha}} C_{\alpha h} q_{\alpha h}(t)
\end{aligned}
$$

where matrices $A_{\alpha}, B_{\alpha}, C_{\alpha}$ represent the nominal matrices and $q_{\alpha h}(t)$ the $h$ th component of vector $q_{\alpha}(t)$ :

$$
q_{\alpha}(t)=\left[\begin{array}{llllll}
q_{\alpha 1}(t) & q_{\alpha 2}(t) & \ldots & q_{\alpha h}(t) & \ldots & q_{\alpha d_{\alpha}}(t)
\end{array}\right]^{T} .
$$

The following additional assumption is required:

- $\Gamma_{\alpha}$ are compact convex sets.

Let the control be obtained by an output feedback control law:

$$
\begin{aligned}
& u_{t}=K_{\alpha} y_{t}=F_{\alpha} x_{t}, \\
& F_{\alpha}=K_{\alpha} C_{\alpha} .
\end{aligned}
$$

Thus, the closed-loop system is given by:

$$
x_{t+1}=A_{\alpha}\left(q_{\alpha}(t)\right) x_{t}+B_{\alpha}\left(q_{\alpha}(t)\right) \operatorname{sat}\left(K_{\alpha} C_{\alpha}\left(q_{\alpha}(t)\right) x_{t}\right) .
$$

Defining the indicator function:

$$
\xi(t):=\left[\xi_{1}(t), \ldots, \xi_{N}(t)\right]^{T},
$$

where $\xi_{i}(t)=1$ if the switching system is in mode $i$ and 0 otherwise, yields the following representation for the closed-loop system:

$$
x_{t+1}=\sum_{i=1}^{N} \xi_{i}(t)\left[A_{i}\left(q_{i}(t)\right) x_{t}+B_{i}\left(q_{i}(t)\right) \operatorname{sat}\left(K_{i} C_{i}\left(q_{i}(t)\right) x_{t}\right] .\right.
$$


Let each convex set $\Gamma_{i}$ has $\mu_{i}$ vertices $v_{i \kappa}, \kappa=1, \ldots, \mu_{i}$ so that for every $q_{i} \in \Gamma_{i}$, one can write $q_{i}=\sum_{\kappa=1}^{\mu_{i}} \beta_{i \kappa} v_{i \kappa}$ with $\sum_{\kappa=1}^{\mu_{i}} \beta_{i \kappa}=1,0 \leq \beta_{i \kappa} \leq 1$. The consequence of this, is that each matrix $A_{i}\left(q_{i}(t)\right), B_{i}\left(q_{i}(t)\right)$ and $C_{i}\left(q_{i}(t)\right)$ can be expressed as a convex combination of the corresponding vertices of the compact set $\Gamma_{i}$ as follows:

$$
\begin{aligned}
& M\left(q_{i}\right):=M_{i}+\sum_{\kappa=1}^{\mu_{i}} \beta_{i \kappa} M\left(v_{i \kappa}\right)=\sum_{\kappa=1}^{\mu_{i}} \beta_{i \kappa} M_{i \kappa} \\
& M\left(v_{i \kappa}\right)=\sum_{h=1}^{d_{i}} M_{i h} v_{i \kappa h}, M_{i \kappa}=M_{i}+M\left(v_{i \kappa}\right), \sum_{\kappa=1}^{\mu_{i}} \beta_{i \kappa}=1,0 \leq \beta_{i \kappa} \leq 1 .
\end{aligned}
$$

where $M_{i}$ represents the nominal matrix. Matrix $M$ can be taken differently as $A, B$ or $C$. Note that the system without uncertainties can be obtained as a particular case of this representation by letting the vertices $v_{i \kappa}=0, \forall i, \forall \kappa$. Besides, equations (69) are directly related to the dimension $d_{i}$ of the convex compact set $\Gamma_{i}$. The saturated uncertain switching system given by (68) can be rewritten as:

$$
x_{t+1}=\sum_{i=1}^{N} \sum_{\kappa=1}^{\mu_{i}} \xi_{i}(t) \beta_{i \kappa}(t)\left[A_{i \kappa} x_{t}+B_{i \kappa} \operatorname{sat}\left(K_{i} C_{i \kappa} x_{t}\right)\right]
$$

The nominal matrices will be represented by $A_{i}, B_{i}$ and $C_{i}$. The nominal system in closed-loop is then given by:

$$
x_{t+1}=\sum_{i=1}^{N} \xi_{i}(t)\left[A_{i} x_{t}+B_{i} \operatorname{sat}\left(K_{i} C_{i} x_{t}\right)\right]
$$

\subsection{Analysis and synthesis of stabilizability}

This section presents sufficient conditions of asymptotic stability of the saturated uncertain switching system given by (69). The synthesis of the controller follows two different approaches, the first one deals firstly with the nominal system and then uses a test to check the asymptotic stability in presence of uncertainties while the second considers the global representation of the uncertain system (69).

Theorem 3.1. If there exist symmetric positive definite matrices $P_{1}, \ldots, P_{N} \in \mathbb{R}^{n \times n}$ and matrices $H_{1}, \ldots, H_{N} \in \mathbb{R}^{m \times n}$ such that

$$
\begin{array}{cc}
{\left[\begin{array}{cc}
P_{i} & {\left[A_{i \kappa}+B_{i \kappa}\left(D_{i s} K_{i} C_{i \kappa}+D_{i s}^{-} H_{i}\right)\right]^{T} P_{j}} \\
* & P_{j}
\end{array}\right]>0,} \\
\forall \kappa=1, \ldots, \mu_{i}, \forall(i, j) \in \mathcal{I}^{2}, \forall s \in[1, \eta],
\end{array}
$$

and

$$
\varepsilon\left(P_{i}, 1\right) \subset \mathcal{L}\left(H_{i}\right),
$$

then the closed-loop uncertain saturated switching system (69) is asymptotically stable $\forall x_{0} \in \Omega:=$ $\bigcup_{i=1}^{N} \varepsilon\left(P_{i}, 1\right)$ and for all switching sequences $\alpha(t)$.

Proof: By using Lemma (2.1), for all $H_{i} \in \mathbb{R}^{m \times n}$ with $\left|H_{i j} x_{t}\right|<1, j \in[1, m]$, where $H_{i j}$ denotes the $j$ th row of matrix $H_{i}$, there exist $\delta_{i \kappa 1} \geq 0, \ldots, \delta_{i \kappa \eta} \geq 0$ such that $\operatorname{sat}\left(K_{i} C_{i \kappa} x_{t}\right)=$ 
$\sum_{s=1}^{\eta} \delta_{i s \kappa}(t)\left[D_{i s} K_{i} C_{i \kappa}+D_{i s}^{-} H_{i}\right] x_{t}, \delta_{i \kappa s}(t) \geq 0, \sum_{s=1}^{\eta} \delta_{i \kappa s}(t)=1$. Then the closed-loop system (69) can be rewritten as

$$
\begin{array}{r}
x_{t+1}=\sum_{s=1}^{\eta} \sum_{i=1}^{N} \sum_{\kappa=1}^{\mu_{i}} \xi_{i}(t) \beta_{i \kappa}(t) \delta_{i \kappa s}(t) A c_{i \kappa s} x_{t} \\
A c_{i \kappa s}:=A_{i \kappa}+B_{i \kappa}\left(D_{i s} K_{i} C_{i \kappa}+D_{i s}^{-} H_{i}\right) .
\end{array}
$$

Consider the Lyapunov function candidate $V(x)=x_{t}^{T}\left(\sum_{i=1}^{N} \xi_{i}(t) P_{i}\right) x_{t}$. Computing its rate of increase along the trajectories of system (69) yields.

$$
\begin{aligned}
\Delta V\left(x_{t}\right) & =x_{t+1}^{T}\left(\sum_{j=1}^{N} \xi_{j}(t+1) P_{j}\right) x_{t+1}-x_{t}^{T}\left(\sum_{i=1}^{N} \xi_{i}(t) P_{i}\right) x_{t} \\
& =x_{t}^{T}\left\{\Sigma^{T}\left(\sum_{j=1}^{N} \xi_{j}(t+1) P_{j}\right) \Sigma-\sum_{i=1}^{N} \xi_{i}(t) P_{i}\right\} x_{t} .
\end{aligned}
$$

where,

$$
\Sigma=\sum_{s=1}^{\eta} \sum_{i=1}^{N} \sum_{\kappa=1}^{\mu_{i}} \xi_{i}(t) \beta_{i \kappa}(t) \delta_{s \kappa i}(t) A c_{i \kappa s}
$$

Let condition (71) be satisfied. For each $i$ and $j$ multiply successively by $\xi_{i}(t), \xi_{j}(t+1), \beta_{i \kappa}(t)$ and $\delta_{i \kappa s}(t)$ and sum. As $\sum_{i=1}^{N} \xi_{i}(t)=\sum_{j=1}^{N} \xi_{j}(t+1)=\sum_{s=1}^{\eta} \delta_{i \kappa s}(t)=\sum_{\kappa=1}^{\mu_{i}} \beta_{i \kappa}(t)=1$, one gets:

$$
\left[\begin{array}{cc}
\sum_{i=1}^{N} \xi_{i}(t) P_{i} & \Pi \\
* & \sum_{j=1}^{N} \xi_{j}(t+1) P_{j}
\end{array}\right]>0
$$

where

$$
\Pi=\Sigma^{T}\left(\sum_{j=1}^{N} \xi_{j}(t+1) P_{j}\right) .
$$

Inequality (75) is equivalent, by Schur complement, to

$$
\Sigma^{T}\left(\sum_{j=1}^{N} \xi_{j}(t+1) P_{j}\right) \Sigma-\sum_{i=1}^{N} \xi_{i}(t) P_{i}<0
$$

Letting $\lambda$ be the largest eigenvalue among all the above matrices, we obtain that

$$
\Delta V\left(x_{t}\right) \leq \lambda x_{t}^{T} x_{t}<0,
$$

which ensures the desired result. Besides, following Theorem 2.4, (71)-(73) also allow for a state belonging to a set $\varepsilon\left(P_{i}, 1\right) \subset \mathcal{L}\left(H_{i}\right)$, before the switch, if a switch occurs at time $t_{k}$, the switch will drive the state to the desired set $\varepsilon\left(P_{j}, 1\right) \subset \mathcal{L}\left(H_{j}\right)$. That means that the set $\Omega$ is a set of asymptotic stability of the uncertain saturated switching system.

Remark 3.1. It is worth to note that the result of Theorem 2.4 can be obtained as a particular case of Theorem 3.1. 
This stability result is now used for control synthesis in two ways: the first consists in computing the controllers only with the nominal system and to test their robustness in a second step; while the second consists in computing in a single step the robust controllers. At this end, the result of Theorem 2.6 can be used to compute matrices $K_{i}, H_{i}$ and $P_{i}$ for the nominal switching system (70). At this step, the stabilizing controllers $K_{i}$ and $H_{i}$ of the nominal system are assumed to be known. Then, the following test has to be performed.

Corollary 3.1. If there exist symmetric positive definite matrices $X_{i}$ such that

$$
\begin{gathered}
{\left[\begin{array}{cc}
X_{i} & \left(A_{i \kappa} X_{i}+B_{i \kappa} D_{i s} K_{i} C_{i \kappa} X_{i}+B_{i \kappa} D_{i s}^{-} H_{i} X_{i}\right)^{T} \\
* & X_{j}
\end{array}\right]>0,} \\
\quad\left[\begin{array}{cc}
1 & \left(H_{i} X_{i}\right)_{l} \\
* & X_{i}
\end{array}\right]>0, \\
\forall(i, j) \in \mathcal{I}^{2}, \quad \forall s \in[1, \eta], \forall l \in[1, m], \forall \kappa \in\left[1, \mu_{i}\right],
\end{gathered}
$$

with $P_{i}=X_{i}^{-1}$, then the closed loop uncertain switching system (69) is asymptotically stable $\forall x_{0} \in$ $\bigcup_{i=1}^{N} \varepsilon\left(P_{i}, 1\right)$ and for all switching sequences $\alpha(t)$.

Proof: The proof is similar to that given for Theorem 2.6.

The second way to deal with robust controller design is to run a global set of LMIs leading, if it is feasible, to the robust controllers directly. However, one can note that this method is computationally more intensive.

Theorem 3.2. If there exist symmetric positive definite matrices $X_{i}$, matrices, $Y_{i}, V_{i}$ and $Z_{i}$ such that

$$
\begin{gathered}
{\left[\begin{array}{cc}
X_{i} & \left(A_{i \kappa} X_{i}+B_{i \kappa} D_{i s} Y_{i} C_{i \kappa}+B_{i \kappa} D_{i s}^{-} Z_{i}\right)^{T} \\
* & X_{j}
\end{array}\right]>0,} \\
{\left[\begin{array}{cc}
1 & Z_{i l} \\
* & X_{i}
\end{array}\right]>0,} \\
V_{i} C_{i \kappa}=C_{i \kappa} X_{i}, \\
\forall(i, j) \in \mathcal{I}^{2}, \quad \forall s \in[1, \eta], \forall l \in[1, m], \forall \kappa \in\left[1, \mu_{i}\right]
\end{gathered}
$$

with

$$
H_{i}=Z_{i} X_{i}^{-1}, K_{i}=Y_{i} V_{i}^{-1}, P_{i}=X_{i}^{-1},
$$

then, the closed-loop uncertain saturated switching system (69) is asymptotically stable $\forall x_{0} \in \Omega$, and for all switching sequences $\alpha(t)$.

Proof: The proof is also similar to that given for Theorem 2.6.

In order to relax the previous LMIs, one can introduce some slack variables as in (Daafouz et al., 2002) and (Benzaouia et al., 2006), as it is now shown:

Theorem 3.3. If there exist symmetric positive definite matrices $X_{i}$, matrices, $Y_{i}, V_{i}, G_{i}$ and $Z_{i}$ such that

$$
\left[\begin{array}{cc}
G_{i}+G_{i}^{T}-X_{i} & \Psi \\
* & X_{j}
\end{array}\right]>0,
$$


with $\Psi=\left(A_{i \kappa} G_{i}+B_{i \kappa} D_{i s} Y_{i} C_{i \kappa}+B_{i \kappa} D_{i s}^{-} Z_{i}\right)^{T}$,

$$
\begin{gathered}
{\left[\begin{array}{cc}
1 & Z_{i l} \\
* & G_{i}+G_{i}^{T}-X_{i}
\end{array}\right]>0,} \\
V_{i} C_{i \kappa}=C_{i \kappa} G_{i}, \\
\forall \kappa=1, \ldots, \mu_{i}, \forall(i, j) \in \mathcal{I}^{2}, \forall s \in[1, \eta], \forall l \in[1, m], \text { with } \\
H_{i}=Z_{i} G_{i}^{-1}, K_{i}=Y_{i} V_{i}^{-1}, P_{i}=X_{i}^{-1} ;
\end{gathered}
$$

then, the closed-loop uncertain saturated switching system (69) is asymptotically stable $\forall x_{0} \in \Omega$ and for all switching sequences $\alpha(t)$.

Proof: The proof is similar to that given for Corollary 2.1

These results can be illustrated with the following example.

Example 3.1. Consider a SISO saturated switching discrete system with two modes given by the following matrices:

$$
\begin{aligned}
& A_{1}\left(q_{1}(t)\right)=\left[\begin{array}{cc}
1 & 1 \\
0 & 1+q_{11}
\end{array}\right] ; B_{1}\left(q_{1}(t)\right)=\left[\begin{array}{l}
10 \\
5
\end{array}\right] ; \\
& C_{1}\left(q_{1}(t)\right)=\left[\begin{array}{cc}
1+q_{12} & 1
\end{array}\right] ; \\
& A_{2}\left(q_{2}(t)\right)= {\left[\begin{array}{cc}
0+q_{21} & -1 \\
0.0001 & 1
\end{array}\right] ; B_{2}\left(q_{2}(t)\right)=\left[\begin{array}{l}
0.5 \\
-2+q_{22}
\end{array}\right] ; } \\
& C_{2}\left(q_{2}(t)\right)=\left[\begin{array}{ll}
2 & 3
\end{array}\right] .
\end{aligned}
$$

The vertices of the domain of uncertainties that affect the first mode are:

$$
\begin{aligned}
& v_{11}=(-0.1,-0.2), \quad v_{12}=(-0.1,0.2) \\
& v_{13}=(0.1,-0.2), \quad v_{14}=(0.1,0.2) .
\end{aligned}
$$

The vertices of the domain of uncertainties that affect the second mode are:

$$
\begin{aligned}
& v_{21}=(-0.2,0.5), \quad v_{22}=(-0.2,-0.1) \\
& v_{23}=(0.3,0.5), \quad v_{24}=(0.3,-0.1) .
\end{aligned}
$$

Using Theorem 2.6, a stabilizing controller for the nominal system is

$$
K_{1}=-0.1000, \quad K_{2}=0.1622 .
$$

To test the robustness, we can use the Corollary 3.1 which leads to the following results:

$$
P_{1}=\left[\begin{array}{cc}
0.0208 & -0.0133 \\
-0.0133 & 0.0257
\end{array}\right] ; P_{2}=\left[\begin{array}{ll}
0.0320 & 0.0023 \\
0.0023 & 0.0474
\end{array}\right]
$$

On the other hand, the use of Theorem 3.2 leads to the following results:

$$
K_{1}=-0.0902, \quad K_{2}=0.1858 .
$$


Figures 5, 6 and 7 concern the first method. In Figure 5, the switching signals $\alpha(t)$ and the evolution of uncertainties used for simulation, are plotted. Figure 6 shows the obtained level set of stability $\bigcup_{i=1}^{N} \varepsilon\left(P_{i}, 1\right)$ which is well contained inside the sets of saturations, while Figure 7 presents some system motions evolving inside the level set starting from different initial states.
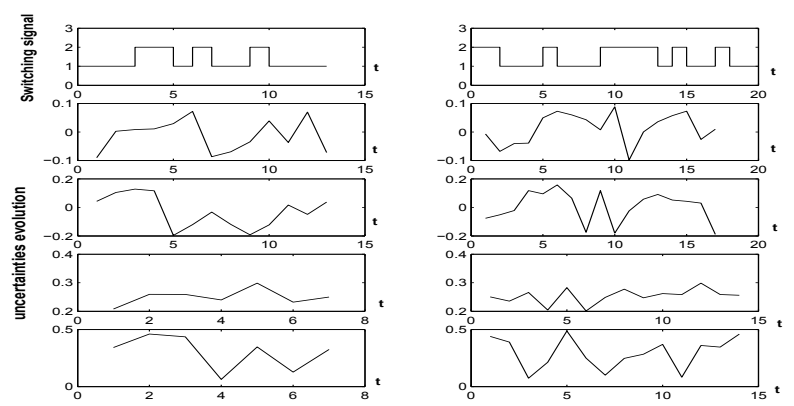

Fig. 5. Switching signals $\alpha(t)$ and uncertainties evolution

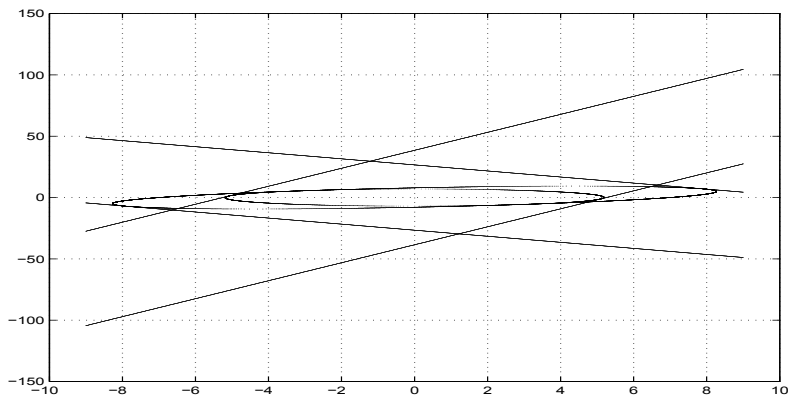

Fig. 6. Inclusion of the ellipsoids inside the polyhedral sets

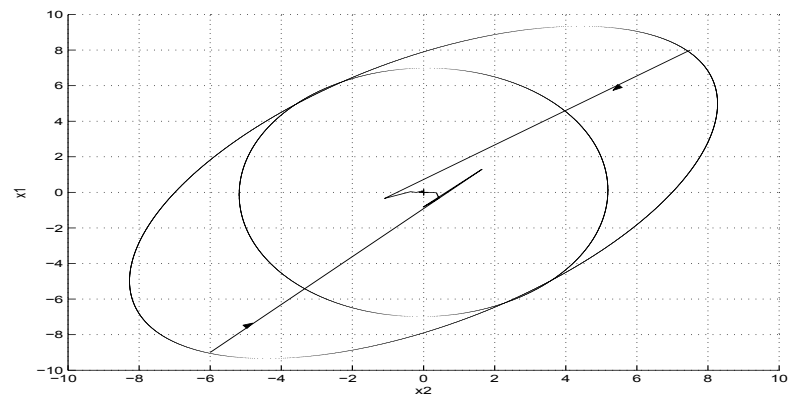

Fig. 7. Motion of the system with controllers obtained with Theorem 2.6 and Corollary 3.1

Figure 7 shows the level set of stability $\bigcup_{i=1}^{N} \varepsilon\left(P_{i}, 1\right)$ using the second method of Theorem 3.2 which is well contained inside the sets of saturations. The use of Theorem 3.3 leads to the following results:

$$
K_{1}=-0.0752, \quad K_{2}=0.1386 ;
$$

Figure 9 shows the level set of stability $\bigcup_{i=1}^{N} \varepsilon\left(P_{i}, 1\right)$ obtained with Theorem 3.3, which is also well contained inside the sets of saturations. 


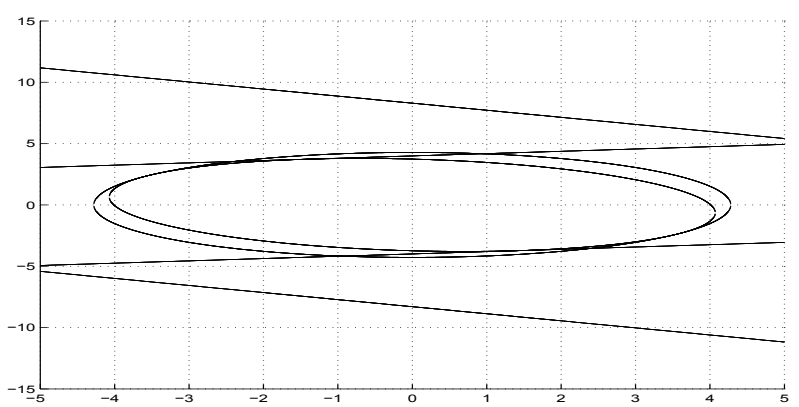

Fig. 8. Inclusion of the ellipsoids inside the polyhedral sets obtained with Theorem 3.2

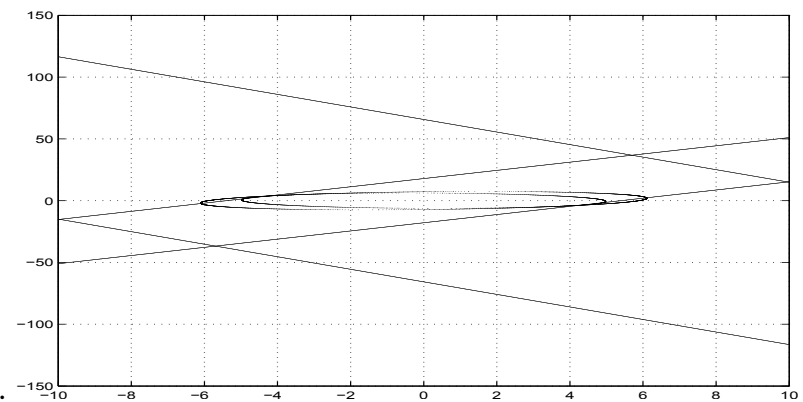

Fig. 9. Inclusion of the ellipsoids inside the polyhedral sets obtained with Theorem 3.3

\subsection{Synthesis of non saturating controllers}

The non saturating controllers who works inside a region of linear behavior can be obtained from the previous results by replacing $D_{s i}=\mathbb{I}$ and $D_{s i}^{-}=0$. The following result presents the synthesis of such controllers.

Theorem 3.4. If there exist symmetric matrices $X_{i}$ and matrices $Y_{i}$ such that

$$
\begin{gathered}
{\left[\begin{array}{cc}
X_{i} & \left(A_{i \kappa} X_{i}+B_{i \kappa} Y_{i} C_{i \kappa}\right)^{T} \\
* & X_{j}
\end{array}\right]>0} \\
{\left[\begin{array}{cc}
1 & Y_{i l} C_{i \kappa} \\
* & X_{i}
\end{array}\right]>0} \\
V_{i} C_{i \kappa}=C_{i \kappa} X_{i}
\end{gathered}
$$

$\forall \kappa=1, \ldots, \mu_{i}, \quad \forall(i, j) \in \mathcal{I}^{2}, \forall l \in[1, m]$, with $, K_{i}=Y_{i} V_{i}^{-1}, P_{i}=X_{i}^{-1}$,

then the uncertain closed-loop switching system (69) is asymptotically stable $\forall x_{0} \in \Omega$ and for all switching sequences $\alpha(t)$.

To illustrate this result, the same system of Example 3.1 is used. Theorem 3.4 leads to the following results:

$$
P_{1}=\left[\begin{array}{cc}
0.2574 & 0 \\
0 & 0.2574
\end{array}\right] ; P_{2}=\left[\begin{array}{ll}
0.2930 & 0.0535 \\
0.0535 & 0.3376
\end{array}\right] ;
$$




$$
K_{1}=-0.0902 ; \quad K_{2}=0.1694
$$

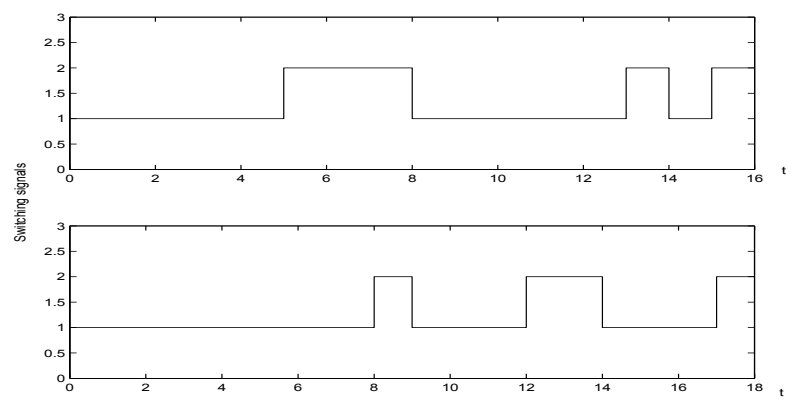

Fig. 10. Switching supervisor signal $\alpha(t)$

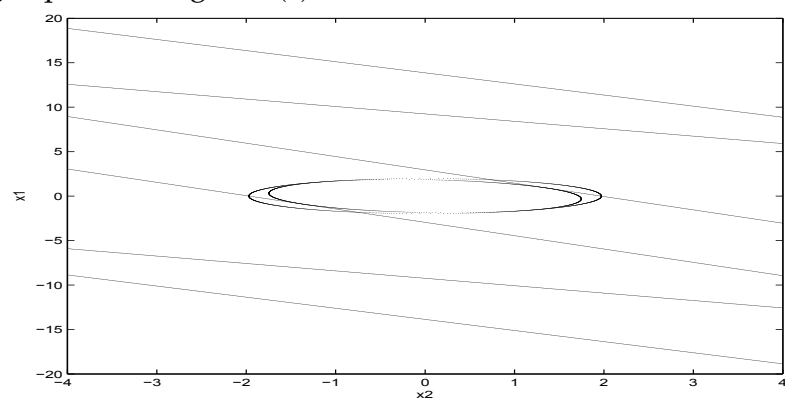

Fig. 11. Inclusion of the ellipsoids obtained with Theorem 3.4

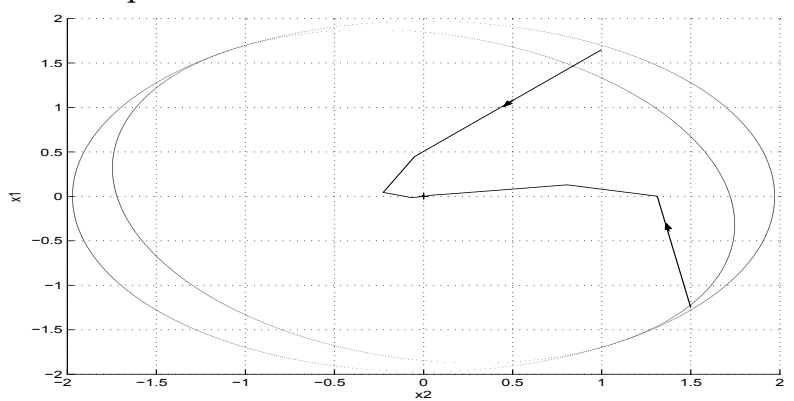

Fig. 12. Motion of the system with controllers obtained with Theorem 3.4

In Figure 5, the evolution of uncertainties is plotted, Figure 10 shows the sequence $\alpha(t)$. The level set $\bigcup_{i=1}^{N} \varepsilon\left(P_{i}, 1\right)$ presented in Figure 11, is also well contained inside the regions of linear behavior. In Figure 12, the trajectories of the system are plotted.

Commennt 3.1. The application of all the proposed results to the same example, shows that the result applied in two steps (Theorem 2.6 and Corollary 3.1) is the least conservative. However, it is worth noting that the result introducing slack variables (Theorem 3.3) is also less conservative even applied in one step. One can expect that this same result applied in two steps can be the less conservative one. 
In this section, two different sufficient conditions of asymptotic stability are obtained for output feedback control of uncertain switching discrete-time linear systems subject to actuator saturations. These conditions allow the synthesis of stabilizing controllers inside a large region of saturation under LMIs formulation. Note that the state feedback control case and the unsaturating controller case can be obtained as particular cases of the study presented in this section. An illustrative example is studied by using the direct resolution of the proposed LMIs. A comparison of the obtained solutions is also given.

\section{Stabilization of saturated switching systems with structured uncertainties}

The objective of this section is to extend the results of (Benzaouia et al., 2006) to uncertain switching systems subject to actuator saturations by using output feedback control. This technique allows to design stabilizing controllers by output feedback for switching discrete-time systems despite the presence of actuator saturations and uncertainties on the system parameters. The case of state feedback control is derived as a particular case. It is also shown that the results obtained in this section with state feedback control are less conservative than those presented in (Yu et al., 2007) where only the state feedback control case is addressed. The main results of this section are published in (Benzaouia et al., 2009c).

\subsection{Problem presentation}

Let us consider the linear uncertain discrete-time switching system described by:

$$
\left\{\begin{array}{l}
x_{t+1}=\mathcal{A}_{\alpha}(t) x_{t}+\mathcal{B}_{\alpha}(t) \operatorname{sat}\left(u_{t}\right) \\
y_{t}=\mathcal{C}_{\alpha}(t) x_{t}
\end{array}\right.
$$

where $x_{t} \in \mathbb{R}^{n}, u_{t} \in \mathbb{R}^{m}$ are the state and the input respectively, sat(.) is the standard saturation, $y_{t} \in \mathbb{R}^{p}$ the output. $\alpha$ is a switching rule taking its values in the finite set $I=\{1, \ldots, N\}$. The saturation function is assumed here to be normalized, i. e., $\mid \operatorname{sat}\left(u_{i} \mid=\min \left(1,\left|u_{i}\right|\right), i=\right.$ $1, \ldots m$.

The system matrices are assumed to be uncertain and satisfy:

$$
\left[\mathcal{A}_{i}(t) \quad \mathcal{B}_{i}(t)\right]=\left[\begin{array}{ll}
A_{i} & B_{i}
\end{array}\right]+M_{i} \Gamma_{i}\left[\begin{array}{ll}
N_{1 i} & N_{2 i}
\end{array}\right]
$$

Let the control be obtained by an output feedback control law:

$$
u_{t}=K_{\alpha} y_{k}=K_{\alpha} C_{\alpha} x_{t}=F_{\alpha} x_{t}
$$

The closed-loop system is given by:

$$
x_{t+1}=\mathcal{A}_{\alpha}(t) x_{t}+\mathcal{B}_{\alpha}(t) \operatorname{sat}\left(K_{\alpha} C_{\alpha} x_{t}\right)
$$

Defining the indicator function:

$$
\xi(t):=\left[\xi_{1}(t), \ldots, \xi_{N}(t)\right]^{T}
$$

where $\xi_{i}(t)=1$ if the switching system is in mode $i$ and 0 otherwise, yields the following representation for the closed-loop system:

$$
x_{t+1}=\sum_{i=1}^{N} \xi_{i}(t)\left[\mathcal{A}_{i}(t) x_{t}+\mathcal{B}_{i}(t) \operatorname{sat}\left(K_{i} C_{i} x_{t}\right)\right]
$$


Assume that there exist $N$ matrices $H_{1}, \ldots, H_{N}$ such that $x(t) \in \mathcal{L}\left(H_{i}\right)$. Using the expression in (10) and rewriting System (94) yields that:

$$
\begin{aligned}
x_{t+1} & =\sum_{s=1}^{\eta} \sum_{i=1}^{N} \xi_{i}(t) \delta_{i s}(t) \mathcal{A} c_{i s}(t) x_{t} \\
\mathcal{A} c_{i s}(t) & :=\mathcal{A}_{i}(t)+\mathcal{B}_{i}(t)\left(D_{i s} K_{i} C_{i}+D_{i s}^{-} H_{i}\right), \quad s \in[1, \eta]
\end{aligned}
$$

\subsection{Analysis and synthesis of stabilizability}

Consider now the saturated uncertain switching system given by (95). The first result synthesizing stabilizing controllers of the uncertain saturated switching system by output feedback is now presented.

Theorem 4.1. If there exist symmetric matrices $X_{1}, \ldots, X_{N}$, matrices $Y_{1}, \ldots, Y_{N}, Z_{1}, \ldots, Z_{N}$, $V_{1}, \ldots, V_{N}$ and a set of real positive scalars $\lambda_{i j s}$, such that

$$
\begin{gathered}
{\left[\begin{array}{ccc}
X_{i} & \Theta_{i s}^{T} & \Phi_{i s}^{T} \\
* & X_{j}-\lambda_{i j s} M_{i} M_{i}^{T} & 0 \\
* & * & \lambda_{i j s} \mathbb{I}
\end{array}\right]>0,} \\
\forall(i, j) \in \mathcal{I} \times \mathcal{I}, \forall s \in[1, \ldots \eta] \\
\quad C_{i} X_{i}=V_{i} C_{i} \\
{\left[\begin{array}{cc}
1 & Z_{i l} \\
* & X_{i}
\end{array}\right]>0,} \\
\forall i \in \mathcal{I}, \forall l \in[1, \ldots m]
\end{gathered}
$$

where $\Theta_{i s}=A_{i} X_{i}+B_{i}\left(D_{i s} Y_{i} C_{i}+D_{i s}^{-} Z_{i}\right)$ and $\Phi_{i s}=N_{1 i} X_{i}+N_{2 i}\left(D_{i s} Y_{i} C_{i}+D_{i s}^{-} Z_{i}\right)$. Then, the uncertain switching system with input saturation in closed-loop (95) with

$$
\begin{aligned}
& K_{i}=Y_{i} V^{-1} \\
& H_{i}=Z_{i} X^{-1}
\end{aligned}
$$

is asymptotically stable $\forall x_{0} \in \Omega=\bigcup_{i=1}^{N} \varepsilon\left(X_{i}^{-1}, 1\right)$ and for all switching sequences $\alpha(t)$.

Proof: By using Lemma 2.1, for all $H_{i} \in \mathbb{R}^{m \times n}$ with $\left|H_{i l} x_{t}\right|<1, l \in[1, m]$, there exist $\delta_{i 1} \geq 0$ $, \ldots, \delta_{i \eta} \geq 0$ such that, $\operatorname{sat}\left(K_{i} C_{i} x_{t}\right)=\sum_{s=1}^{\eta} \delta_{i s}(t)\left[D_{i s} K_{i} C_{i}+D_{i s}^{-} H_{i}\right] x_{t}, \delta_{i s}(t) \geq 0, \sum_{s=1}^{\eta} \delta_{i s}(t)=1$. System (94) is then rewritten as (95).

Consider the Lyapunov function candidate $V(x)=x_{t}^{T}\left(\sum_{i=1}^{N} \xi_{i}(t) P_{i}\right) x_{t}$. Computing its rate of increase along the trajectories of system (95) yields:

$$
\begin{aligned}
\Delta V\left(x_{t}\right) & =x_{t+1}^{T}\left(\sum_{j=1}^{N} \xi_{j}(t+1) P_{j}\right) x_{t+1}-x_{t}^{T}\left(\sum_{i=1}^{N} \xi_{i}(t) P_{i}\right) x_{t} \\
& =\sum_{s=1}^{\eta} \sum_{j=1}^{N} \xi_{j}(t+1) \delta_{i s} x_{t}^{T}\left[\mathcal{A}_{i}+\mathcal{B}_{i}\left(D_{i s} F_{i}+D_{i s}^{-} H_{i}\right)\right]^{T} P_{j}\left[\mathcal{A}_{i}\right. \\
& \left.+\mathcal{B}_{i}\left(D_{i s} F_{i}+D_{i s}^{-} H_{i}\right)\right] x_{t}-\sum_{i=1}^{N} \xi_{i}(t) x_{t}^{T} P_{i} x_{t}
\end{aligned}
$$


Since, $\sum_{s=1}^{\eta} \delta_{i s}(t)=\sum_{j=1}^{N} \xi_{j}(t+1)=\sum_{i=1}^{N} \xi_{i}(t)=1$, one should obtain

$$
\begin{aligned}
\Delta V\left(x_{t}\right)= & \sum_{j=1}^{N} \sum_{i=1}^{N} \sum_{s=1}^{\eta} \xi_{i}(t) \xi_{j}(t+1) \delta_{i s}(t) x_{t}^{T} \\
& \left(\left[\mathcal{A}_{i}+\mathcal{B}_{i}\left(D_{i s} F_{i}+D_{i s}^{-} H_{i}\right)\right]^{T} P_{j}\left[\mathcal{A}_{i}+\mathcal{B}_{i}\left(D_{i s} F_{i}+D_{i s}^{-} H_{i}\right)\right]-P_{i}\right) x_{t}
\end{aligned}
$$

A sufficient condition to obtain $\Delta V\left(x_{t}\right)<0$ is that:

$$
\left[\mathcal{A}_{i}+\mathcal{B}_{i}\left(D_{i s} F_{i}+D_{i s}^{-} H_{i}\right)\right]^{T} P_{j}\left[\mathcal{A}_{i}+\mathcal{B}_{i}\left(D_{i s} F_{i}+D_{i s}^{-} H_{i}\right)\right]-P_{i}=-\Psi_{s i j}<0
$$

By applying Schur complement to (101), the following equivalent inequality is obtained:

$$
\left[\begin{array}{cc}
P_{i} & {\left[\mathcal{A}_{i}+\mathcal{B}_{i}\left(D_{i s} K_{i} C_{i}+D_{i s}^{-} H_{i}\right)\right]^{T}} \\
* & P_{j}^{-1}
\end{array}\right]>0,
$$

Letting $X_{i}=P_{i}^{-1}, Y_{i}=K_{i} V_{i}, C_{i} X_{i}=V_{i} C_{i}, Z_{i}=H_{i} X_{i}$ and multiplying the above inequality on both sides by $\operatorname{diag}\left(X_{i}, \mathbb{I}\right)$ we get

$$
\left[\begin{array}{cc}
X_{i} & {\left[\mathcal{A}_{i} X_{i}+\mathcal{B}_{i}\left(D_{i s} K_{i} C_{i}+D_{i s}^{-} H_{i}\right) X_{i}\right]^{\top}} \\
* & X_{j}
\end{array}\right]>0,
$$

Taking account of (91), inequality (103) can be developed as follows:

$$
\begin{aligned}
& -\left[\begin{array}{cc}
X_{i} & {\left[A_{i} X_{i}+B_{i}\left(D_{i s} Y_{i} C_{i}+D_{i s}^{-} Z_{i}\right)\right]^{\top}} \\
* & X_{j}
\end{array}\right]+\left[\begin{array}{c}
{\left[N_{1 i} X_{i}+N_{2 i}\left(D_{i s} Y_{i} C_{i}+D_{i s}^{-} Z_{i}\right)\right]^{\top}} \\
0
\end{array}\right] \\
& \Gamma_{i}^{\top}\left[\begin{array}{ll}
0 & -M_{i}^{T}
\end{array}\right]+\left[\begin{array}{c}
0 \\
-M_{i}
\end{array}\right] \Gamma_{i}\left[\left[N_{1 i} X_{i}+N_{2 i}\left(D_{i s} Y_{i} C_{i}+D_{i s}^{-} Z_{i}\right)\right] \quad 0\right]<0,
\end{aligned}
$$

by virtue of Lemma2.2, this inequality holds if and only if there exist positive scalars $\lambda_{i j s}$ such that

$$
\begin{array}{r}
-\left[\begin{array}{cc}
X_{i} & \Theta_{i s}^{T} \\
* & X_{j}
\end{array}\right]+\lambda_{i j s}\left[\begin{array}{c}
0 \\
-M_{i}
\end{array}\right]\left[\begin{array}{ll}
0 & -M_{i}^{T}
\end{array}\right]+\frac{1}{\lambda_{i j s}}\left[\begin{array}{ll}
\Phi_{i s} & 0
\end{array}\right]\left[\begin{array}{c}
\Phi_{i s}^{T} \\
0
\end{array}\right]<0, \\
\forall(i, j) \in \mathcal{I} \times \mathcal{I}, \forall s \in[1, \ldots \eta] .
\end{array}
$$

Or in a compact form,

$$
\begin{array}{cc}
{\left[\begin{array}{cc}
X_{i}-\frac{1}{\lambda_{i j s}} \Phi_{i s} \Phi_{i s}^{T} & \Theta_{i s}^{T} \\
* & X_{j}-\lambda_{i j s} M_{i} M_{i}^{T}
\end{array}\right]>0,} \\
\\
\quad \forall(i, j) \in \mathcal{I} \times \mathcal{I}, \forall s \in[1, \eta]
\end{array}
$$

where $\Phi_{i s}$ and $\Theta_{i s}$ are defined before.

By Schur complement, inequality (104) is equivalent to (96). One can then bound the rate of increase as follows,

$$
\begin{array}{r}
\Delta V\left(x_{t}\right) \leq-\gamma\left(\left\|x_{t}\right\|\right) \\
\gamma\left(\left\|x_{t}\right\|\right)=\min _{i j s} \lambda_{\text {min }}\left(\Psi_{i j s}\right)\left\|x_{t}\right\|^{2} .
\end{array}
$$


Using (Hu et al., 2002), the inclusion condition (29) can also be transformed to the equivalent LMI (98) by virtue of the results of (Boyd et al., 1994).

To obtain larger ellipsoid domains $\varepsilon\left(P_{i}, 1\right)$, we can use a shape reference set $\mathcal{X}_{R} \subset \mathbb{R}^{n}$, in terms of a polyhedron or ellipsoid to measure the size of the domain of attraction.

For a set $\mathcal{L} \subset \mathbb{R}^{n}$ which contains the origin, define $\mu\left(\mathcal{X}_{R}, \mathcal{L}\right)=\sup \left\{\mu>0, \mu \mathcal{X}_{R} \subset \mathcal{L}\right\}$. Here, we choose $\mathcal{X}_{R}$ to be a polyhedral defined as $\mathcal{X}_{R}=c o\left\{\omega_{1}, \omega_{2}, \ldots, \omega_{q}\right\}$, where $\omega_{1}, \omega_{2}, \ldots, \omega_{q}$ are a prior given points in $\mathbb{R}^{n}$.

The problem can be formulated as the following constrained optimization problem

$$
(P b .4):\left\{\begin{array}{c}
\max _{X_{i}>0, Y_{i}, Z_{i}, \lambda_{i j s}}\left(\mu_{i}\right) \\
\text { s.t. } \mu \mathcal{X}_{R} \subset \varepsilon\left(P_{i}, 1\right) \\
(96),(98), \\
i=1, \ldots, N
\end{array}\right.
$$

As is explained in (Hu et al., 2001) and ( $\mathrm{Hu}$ and Lin, 2002), the constraint $\mu \mathcal{X}_{R} \subset \varepsilon\left(P_{i}, 1\right)$ is satisfied if the following matrix inequalities hold:

$$
\begin{aligned}
& {\left[\begin{array}{cc}
\mu_{i}^{-2} & \omega_{l}^{T} \\
\omega_{l} & X_{i}
\end{array}\right] \geq 0,} \\
& \forall i \in \mathcal{I}, \quad \forall l \in[1, q]
\end{aligned}
$$

The problem of enlarging the domain of attraction can be reduced to an LMI optimization problem defined as follows:

$$
(P b .5):\left\{\begin{array}{c}
\min _{X_{i}>0, Y_{i}, Z_{i}, \lambda_{i j s}}\left(\gamma_{i}\right) \\
\text { s.t. }(96),(98),(105) \\
i=1, \ldots, N
\end{array}\right.
$$

where $\gamma_{i}=\mu_{i}^{-2}$.

Commennt 4.1. The results of Theorem 4.1 applies directly to switching systems with state feedback control by taking $C_{i}=\mathbb{I}$. In this case, these results can be compared to the one given in (Yu et al.,2007). The fact that the scalars $\lambda_{i j s}$ are all kept equal in (Yu et al.,2007), makes the result obviously more conservative. An example will show this conservatism.

In order to more improve the result of Theorem 4.1 by introducing additional slack variables, the following corollary is presented.

Corollary 4.1. If there exist symmetric matrices $X_{i}>0$, matrices $G_{i}, Y_{i}, V_{i}, Z_{i}$ and positive scalars $\lambda_{i j s}$ such that

$$
\begin{gathered}
{\left[\begin{array}{cccc}
G_{i}^{T}+G_{i}-X_{i} & Y_{i s}^{T} & 0 & \Lambda_{i s}^{T} \\
* & X_{j} & \lambda_{i j s} M_{i} & 0 \\
* & * & \lambda_{i j s} I & 0 \\
* & * & * & \lambda_{i j s} \mathbb{I}
\end{array}\right]>0} \\
\forall(i, j) \in \mathcal{I}^{2}, \forall s \in[1, \ldots \eta]
\end{gathered}
$$




$$
\begin{array}{r}
C_{i} G_{i}=V_{i} C_{i} \\
{\left[\begin{array}{cc}
1 & Z_{i l} \\
* & G_{i}^{T}+G_{i}-X_{i}
\end{array}\right]>0,} \\
\forall i \in \mathcal{I}, \forall s \in[1, \ldots \eta], \forall l \in[1, \ldots m]
\end{array}
$$

where $Y_{i s}=A_{i} G_{i}+B_{i}\left(D_{i s} Y_{i} C_{i}+D_{i s}^{-} Z_{i}\right)$ and $\Lambda_{i s}=N_{1 i} G_{i}+N_{2 i}\left(D_{i s} Y_{i} C_{i}+D_{i s}^{-} Z_{i}\right)$.

Then, the uncertain switching system with input saturation in the closed-loop (95) with

$$
\begin{aligned}
K_{i} & =Y_{i} V^{-1} \\
H_{i} & =Z_{i} G^{-1}
\end{aligned}
$$

is asymptotically stable $\forall x_{0} \in \Omega=\bigcup_{i=1}^{N} \varepsilon\left(X_{i}^{-1}, 1\right)$ and for all switching sequences $\alpha(t)$.

Proof: It was proven in (Benzaouia et al., 2004) and (Benzaouia et al., 2006) that condition (102) is feasible if and only if there exists non singular matrices $G_{i}$ such that the following inequality holds:

$$
\begin{array}{rr}
G_{i}+G_{i}^{T}-X_{i} & G_{i}^{T}\left[\mathcal{A}_{i}+\mathcal{B}_{i}\left(D_{i s} K_{i} C_{i}+D_{i s}^{-} H_{i}\right)\right]^{T} \\
* & X_{j} \\
\forall(i, j) & \in \mathcal{I} \times \mathcal{I}, \forall s \in[1, \eta]
\end{array}
$$

where $X_{i}=P_{i}^{-1}$. The same reasoning is then followed as in the proof of Theorem 4.1 leading to (106). Inequality (108) was also proven in (Benzaouia et al., 2006) by using (Boyd et al., 1994).

These results can be illustrated with the following example.

Example 4.1. Consider a SISO saturated switching discrete-time system with two modes given by the following matrices:

$$
\begin{aligned}
& A_{1}=\left[\begin{array}{ll}
1 & 1 \\
0 & 1
\end{array}\right], B_{1}=\left[\begin{array}{c}
10 \\
5
\end{array}\right], M_{1}=0.1 \mathrm{II}, N_{11}=N_{12}=0.01 \mathrm{II} \\
& A_{2}=\left[\begin{array}{cc}
0 & -1 \\
0 & 1
\end{array}\right], B_{2}=\left[\begin{array}{c}
0.5 \\
-2
\end{array}\right], M_{2}=0.1 \mathrm{II}, N_{21}=N_{22}=0.01 \mathrm{II}
\end{aligned}
$$

By solving the optimization problem (Pb.5) for the above system, we can obtain the following results:

$$
\begin{aligned}
& P_{1}=10 E-03\left[\begin{array}{ll}
4.3324 & 1.2516 \\
1.2516 & 4.3324
\end{array}\right] ; P_{2}=10 E-03\left[\begin{array}{ll}
4.3988 & 2.0934 \\
2.0934 & 6.1433
\end{array}\right], \\
& H_{1}=[-0.0261536-0.0653823] ; H_{2}=[-0.00001920 .0717335] \\
& K_{1}=-0.1000089 ; K_{2}=0.1256683
\end{aligned}
$$

The corresponding figures are given by Figure13 and Figure 14. By applying Corollary 4.1, the following results are obtained:

$$
P_{1}=10 E-03\left[\begin{array}{ll}
1.0047 & 0.1917 \\
0.1917 & 2.0626
\end{array}\right] ; P_{2}=10 E-04\left[\begin{array}{cc}
7.380 & 1.823 \\
1.823 & 23.530
\end{array}\right],
$$




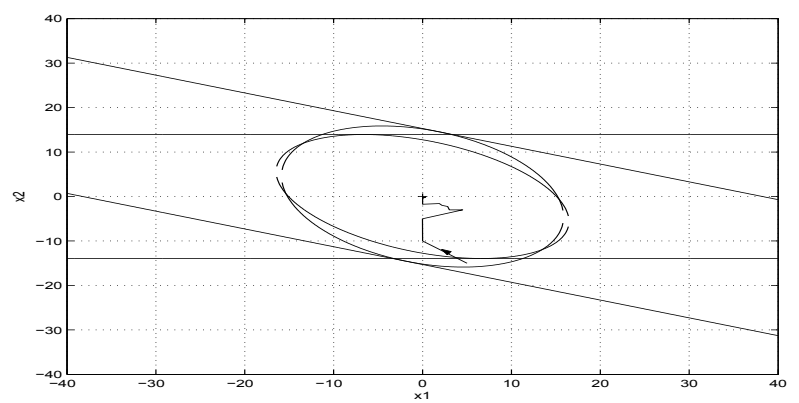

Fig. 13. Inclusion of the ellipsoids inside the polyhedral sets using Theorem 4.1

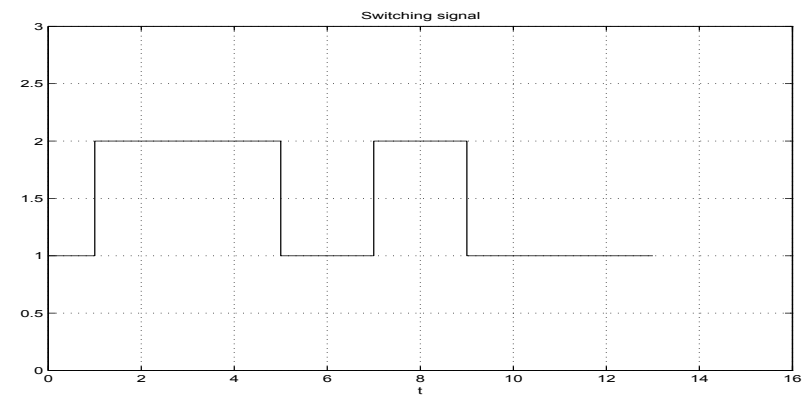

Fig. 14. The switching sequences

$$
\begin{aligned}
& H_{1}=[-0.0179753-0.0405034] ; H_{2}=\left[\begin{array}{lll}
-0.0000072 & 0.0480397
\end{array}\right] \\
& K_{1}=-0.0597163 ; K_{2}=0.0365389 .
\end{aligned}
$$

The corresponding figures are given by Figure 15 and Figure 16. In order to compare the present results with (Yu et al., 2007) for state feedback control, the same example is considered. The results of Theorem 4.1 give:

$$
\begin{gathered}
P_{1}=10 E-08\left[\begin{array}{cc}
8.656 & 60 \\
60 & 6900
\end{array}\right] \quad P_{2}=10 E-08\left[\begin{array}{cc}
1.691 & 7.501 \\
7.501 & 6750
\end{array}\right], \\
H_{1}=[-0.0002470-0.0060812] ; H_{2}=\left[\begin{array}{ll}
-0.0000081 & 0.0081436
\end{array}\right] \\
F_{1}=[-0.0003254-0.0099873] ; F_{2}=\left[\begin{array}{ll}
-0.0068339 & 0.6156659
\end{array}\right]
\end{gathered}
$$

while the results of (Yu et al., 2007) give:

$$
\begin{aligned}
P_{1} & =10 E-07\left[\begin{array}{cc}
2 & 8 \\
8 & 773
\end{array}\right] ; P_{2}=10 E-08\left[\begin{array}{cc}
4.894 & 20 \\
20 & 7630
\end{array}\right], \\
H_{1} & =[-0.0003203-0.0066782] ; H_{2}=\left[\begin{array}{ll}
-0.0000016 & 0.0086701
\end{array}\right] \\
F_{1} & =[-0.0050262-0.3821976] ; F_{2}=[-0.01128930 .3695061]
\end{aligned}
$$

The corresponding level sets are depicted in Figure 17 and Figure 18 where the conservatism of the results of (Yu et al., 2007) is obvious. 


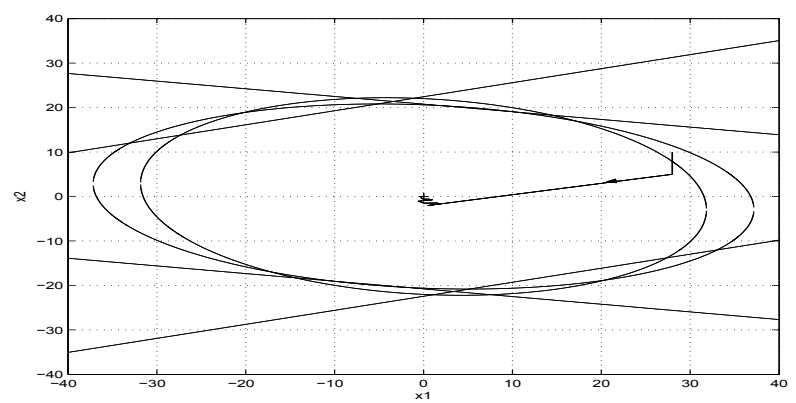

Fig. 15. Inclusion of the ellipsoids inside the polyhedral sets using Theorem 4.1

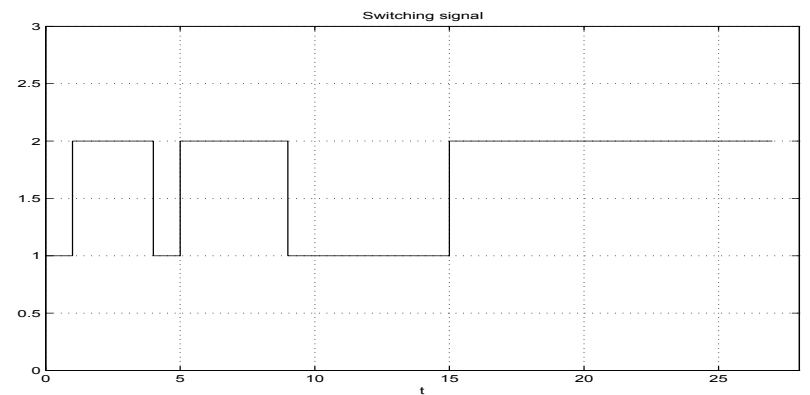

Fig. 16. The switching sequences

This section studied uncertain switching systems with output feedback control which extends the results of (Yu et al., 2007) given with state feedback control. In order to compare these two results, a numerical study using only the particular case of the present work, as mentioned by the Comment 4.1, is also presented. The obtained improvements with the methods presented in this work are shown in Figure 17 and Figure 18. A numerical example is used to illustrate all these techniques.

\section{CONCLUSION}

In this chapter, two main different sufficient conditions of asymptotic stability are obtained for switching discrete-time linear systems subject to actuator saturations for each case: state feedback and output feedback control. The first allows the synthesis of stabilizing controllers inside a large region of linear behavior while the second applies the idea of Lemma (Hu et al., 2002) which rewrites the saturation function under a combination of $2^{m}$ elements to obtain stabilizing controllers tolerating saturations to take effect. A particular attention is given to the output feedback case which has additive complexity due to the output equation. In this sense, three different LMIs are presented for this case. The main results of this work are given under LMIs formulation leading to the design of the stabilizing state feedback and output feedback controllers for the system. Even the dynamical system is a switching system, it is shown that the set $\Omega$ formed by the union of all the ellipsoid level sets associated to each subsystem, constitutes a set of asymptotic stability. The first time that this important result is established for saturated switching systems is in (Benzaouia et al., 2006). Two illustrative examples are studied by using the solution of the proposed LMIs. A comparison of the obtained 


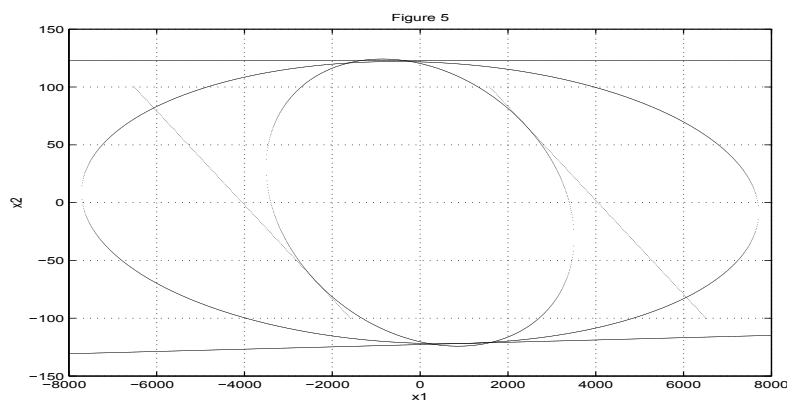

Fig. 17. Inclusion of the ellipsoids inside the polyhedral sets using Theorem 4.1

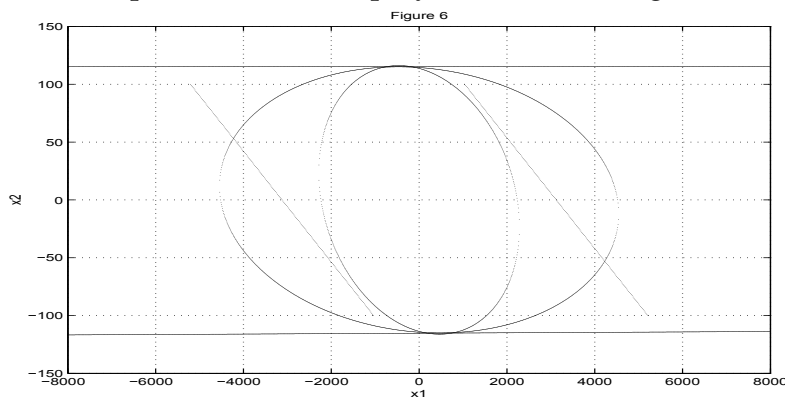

Fig. 18. Inclusion of the ellipsoids inside the polyhedral sets using (Yu et al., 2007)

solutions is also given.

Further, sufficient conditions of stabilization of switching linear discrete-time systems with polytopic and structured uncertainties are also obtained. These conditions are given under LMIs form. Both the cases of feedback control and output control are studied for polytopic uncertainties. However, for structured uncertainties, the output feedback control is presented extending the results of (Yu et al., 2007) given with state feedback control. A comparison study is given with a numerical particular case. The obtained improvements with our method are also shown. A numerical example is used to illustrate all these techniques. As a perspective, two new works developed for switching systems without saturation, the first concerns positive switching systems (Benzaouia and Tadeo, 2008) while the second concerns the output feedback problem (Bara and Boutayeb, 2006) can be used with saturated controls.

\section{REFERENCES}

G. I. Bara and M. Boutayeb, "Switched output feed back stabilization of discrete-time switched systems". $45^{\text {th }}$ Conference on Decision and Control, December 13-15, San Diego, pp. 26672672, 2006.

A. Benzaouia, C. Burgat, "Regulator problem for linear discrete-time systems with nonsymmetrical constrained control". Int. J. Control. Vol. 48, N 6, pp. 2441-2451, 1988.

A. Benzaouia, A. Hmamed, "Regulator Problem for Linear Continuous Systems with Nonsymmetrical Constrained Control". IEEE Trans. Aut. Control, Vol. 38, $N^{\circ} 10$, pp. 1556-1560, 1993. 
A. Benzaouia and A. Baddou, "Piecwise linear constrained control for continuous time systems". IEEE Trans. Aut. Control, Vol. 44, N 7 pp. 1477-1481, 1999.

A. Benzaouia, A. Baddou and S. Elfaiz, "Piecewise linear constrained control for continuoustime systems: An homothetic expansion method of the initial domain. Journal of Dynamical and Control Systems. Vol. 12, N 2 (April), pp. 277-287, 2006.

A. Benzaouia, L. Saydy and O. Akhrif, "Stability and control synthesis of switched systems subject to actuator saturation". American Control Conference, June 30- July 2, Boston, 2004.

A. Benzaouia, O. Akhrif and L. Saydy, "Stability and control synthesis of switched systems subject to actuator saturation by output feedback". $45^{\text {th }}$ Conference on Decision and Control, December 13-15, San Diego, 2006.

A. Benzaouia, F. Tadeo and F. Mesquine, "The Regulator Problem for Linear Systems with Saturations on the Control and its Increments or Rate: An LMI approach". IEEE Transactions on Circuit and Systems Part I, Vol. 53, Nº. 12, pp. 2681-2691, 2006.

A. Benzaouia, E. DeSantis, P. Caravani and N. Daraoui, "Constrained Control of Switching Systems: A Positive Invariance Approach". Int. J. of Control, Vol. 80, Issue 9, pp. 1379-1387, 2007.

A. Benzaouia and F. Tadeo. "Output feedback stabilization of positive switching linear discrete-time systems". 16 ${ }^{\text {th }}$ Mediterranean Conference, Ajaccio, France June 25-27, 2008.

A. Benzaouia, O. Akhrif and L. Saydy. "Stabilitzation and Control Synthesis of Switching Systems Subject to Actuator Saturation". Int. J. Systems Sciences. To appear 2009.

A. Benzaouia, O. Benmesaouda and Y. Shi " Output feedback Stabilization of uncertain saturated discrete-time switching systems". IJICIC. Vol. 5, $N^{\circ}$. 6, pp. 1735-1745, 2009.

A. Benzaouia, O. Benmesaouda and F. Tadeo. "Stabilization of uncertain saturated discretetime switching systems". Int. J. Control Aut. Sys. (IJCAS). Vol. 7, N. 5, pp. 835-840, 2009.

F. Blanchini, "Set invariance in control - a survey". Automatica, Vol. 35, N . 11, pp. 1747-1768, 1999.

F. Blanchini and C. Savorgnan, "Stabilizability of switched linear systems does not imply the existence of convex Lyapunov functions". $45^{\text {th }}$ Conference on Decision and Control, December 13-15, San Diego, pp. 119-124, 2006.

F. Blanchini, S. Miani and F. Mesquine, "A Separation Principle for Linear Switching Systems and Parametrization of All Stabilizing Controllers, "IEEE Trans. Aut. Control", Vol. 54, No. 2, pp. 279-292, 2009.

E. L. Boukas, A. Benzaouia. "Stability of discrete-time linear systems with Markovian jumping parameters and constrained Control". IEEE Trans. Aut. Control. Vol. 47, $N^{\circ}$. 3, pp. 516-520, 2002.

S. P. Boyd, EL Ghaoui, E. Feron, and V. Balakrishnan. "Linear Matrix Inequalities in System and Control Theory". SIAM, Philadelphia, PA, 1994.

M. S. Branicky, "Multiple Lyapunov functions and other analysis tools for switched and hybrid systems". IEEE Automat. Contr., Vol. 43, pp. 475-482, 1998.

E. F. Camacho and C.Bordons, "Model Predictive Control"', Springer-Verlag, London, 2004.

M. Chadli, D. Maquin and J. Ragot. "An LMI formulation for output feedback stabilization in multiple model approach". In Proc. of the 41 th CDC, Las Vegas, Nevada, 2002.

J. Daafouz and J. Bernussou. "Parameter dependent Lyapunov functions for discrete-time systems with time varying parametric uncertainties", Systems and Control Letters, Vol. 43, No. 5, pp. 355-359, 2001. 
J. Daafouz, P. Riedinger and C. Iung. "Static output feedback control for switched systems". Procceding of the 40th IEEE Conference on Decision and Control, Orlando, USA, 2001.

J. Daafouz, P. Riedinger and C. Iung. "Stability analysis and control synthesis for switched systems: a switched Lyapunov function approach". IEEE Trans. Aut. Control, Vol. 47, $N^{\circ} .11$, pp. 1883-1887, 2002.

L. El Ghaoui, F. Oustry, and M. AitRami "A Cone Complementarity Linearization Algorithm for Static Output-Feedback and Related Problems". IEEE Trans. Aut. Control, Vol.42, $N^{\circ} 8, p p .1171-1176,1997$.

L. Hetel, J. Daafouz, and C. Iung. "Stabilization of Arbitrary Switched Linear Systems With Unknown Time-Varying Delays". IEEE Trans. Aut. Control, Vol. 51, $N^{\circ}$. 10, pp. 1668-1674, 2006.

G. Ferrai-Trecate, F. A. Cuzzola, D. Mignone and M. Morari. "Analysis and control with performanc of piecewise affine and hybrid systems". Procceding of the American Control Conference, Arlington, USA, 2001.

P. Gutman and P. Hagandar. "A new design of constrained controllers for linear systems," IEEE Trans. Aut. Cont., Vol.AC - 30, pp.22 - 33, 1985.

T. Hu, Z. Lin and B. M. Chen, "An analysis and design method for linear systems subject to actuator saturation and disturbance". Automatica, Vol. 38, pp. 351-359, 2002.

T. Hu, Z. Lin, "Control Systems with Actuator Saturation: Analysis and Design", BirkhVauser, Boston, 2001.

T. Hu, L. Ma and Z. Lin. "On several composite quadratic Lyapunov functions for switched systems". Procceding of the $45^{t} h$ IEEE Conference on Decision and Control, San Diego, USA, pp. 113-118, 2006.

D. Liberzon, "Switching in systems and control". Springer, 2003.

J. Lygeros, C. Tomlin, and S. Sastry. "Controllers for reachability specifications for hybrid systems", Automatica, vol. 35, 1999.

D. Mignone, G. Ferrari-Trecate,and M. Morari, "Stability and stabilization of Piecwise affine and hybrid systems: an LMI approach". Procceding of the 39th IEEE Conference on Decision and Control, Sydney, Australia, 2000.

E. F. Mulder, M. V. Kothare and and M. Morari, "Multivariable anti-windup controller synthesis using linear matrix inequalities". Automatica, Vol. 37, No.9, pp. 1407-1416, 2001.

R. N. Shorten, and Narendra K.S, a) "On the existence of a commun Lyapunov function for linear stable switching systems". Proc. 10th, Yale Workshop on Adaptive and Learning Systems, pp.130-140, 1998. b) "A sufficient condition for the existence of a commun Lyapunov function for two second-orderliinear systems". Proc, 36th Conf. Decision and Control, pp. 3521-3522,1997.

D. Xie, L. Wang, F. Hao, G. Xie, "Robust Stability Analysis and Control Synthesis for Discrettime Uncertain switched Systems". Conference on Decision and control. Hawaii, USA, 2003.

J. Yu, G. Xie and L. Wang, "Robust Stabilization of discrete-time switched uncertain systems subject to actuator saturation". American Control Conference, New York, July 11-13, 2007. 


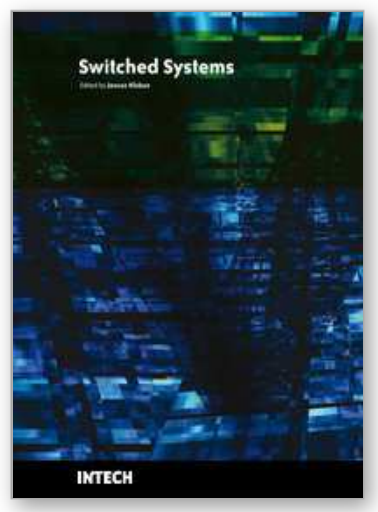

\author{
Switched Systems \\ Edited by Janusz Kleban
}

ISBN 978-953-307-018-6

Hard cover, 174 pages

Publisher InTech

Published online 01, December, 2009

Published in print edition December, 2009

This book presents selected issues related to switched systems, including practical examples of such systems. This book is intended for people interested in switched systems, especially researchers and engineers.

Graduate and undergraduate students in the area of switched systems can find this book useful to broaden their knowledge concerning control and switching systems.

\title{
How to reference
}

In order to correctly reference this scholarly work, feel free to copy and paste the following:

Abdellah Benzaouia (2009). Stabilization of saturated switching systems, Switched Systems, Janusz Kleban (Ed.), ISBN: 978-953-307-018-6, InTech, Available from: http://www.intechopen.com/books/switchedsystems/stabilization-of-saturated-switching-systems

\section{INTECH}

open science | open minds

\section{InTech Europe}

University Campus STeP Ri

Slavka Krautzeka 83/A

51000 Rijeka, Croatia

Phone: +385 (51) 770447

Fax: +385 (51) 686166

www.intechopen.com

\section{InTech China}

Unit 405, Office Block, Hotel Equatorial Shanghai

No.65, Yan An Road (West), Shanghai, 200040, China 中国上海市延安西路65号上海国际贵都大饭店办公楼 405 单元

Phone: +86-21-62489820

Fax: $+86-21-62489821$ 
(C) 2009 The Author(s). Licensee IntechOpen. This chapter is distributed under the terms of the Creative Commons Attribution-NonCommercialShareAlike-3.0 License, which permits use, distribution and reproduction for non-commercial purposes, provided the original is properly cited and derivative works building on this content are distributed under the same license. 